\title{
Quiet-Sun inter-network magnetic fields observed in the infrared ${ }^{\star}$
}

\author{
E. V. Khomenko ${ }^{1}$, M. Collados ${ }^{2}$, S. K. Solanki ${ }^{3}$, A. Lagg ${ }^{3}$, and J. Trujillo Bueno ${ }^{2, \star \star ~}$ \\ 1 Main Astronomical Observatory, NAS, 03680 Kyiv, Zabolotnogo str. 27, Ukraine \\ 2 Instituto de Astrofísica de Canarias, 38200, La Laguna, Tenerife, Spain \\ e-mail: mcv@ll.iac.es; jtb@ll.iac.es \\ 3 Max-Planck-Institut für Aeronomie, 37191 Katlenburg-Lindau, Germany \\ e-mail: solanki@linmpi.mpg.de; lagg@linmpi.mpg.de
}

Received 22 November 2002 / Accepted 15 April 2003

\begin{abstract}
This paper presents the results of an investigation of the quiet Sun's magnetic field based on high-resolution infrared spectropolarimetric observations obtained with the Tenerife Infrared Polarimeter (TIP) at the German VTT of the Observatorio del Teide. We observed two very quiet regions at disc centre. The seeing was exceptionally good during both observing runs, being excellent during one of them. In both cases the network was intentionally avoided to the extent possible, to focus the analysis on the characteristics of the weak polarization signals of the inter-network regions. We find that the Stokes $V$ profile of Fe I $15648 \AA$ line in almost 50\% of the pixels and Stokes $Q$ and/or $U$ in $20 \%$ of the pixels have a signal above $10^{-3}$ (in units of continuum intensity $I_{\mathrm{c}}$ ), which is significantly above the noise level of $2-3 \times 10^{-4}$. This implies that we detect fluxes as low as $2 \times 10^{15} \mathrm{Mx} / \mathrm{px}$. We find evidence that we have detected most of the net flux that is in principle detectable at $1^{\prime \prime}$ resolution with the Zeeman effect. The observed linear polarization resulting from the transverse Zeeman effect indicates that the magnetic fields have a broad range of inclinations, although most of the pixels show polarization signatures which imply an inclination of about $20^{\circ}$. Nearly $30 \%$ of the selected $V$-profiles have irregular shapes with 3 or more lobes, suggesting mixed polarities with different LOS velocity within the resolution element. The profiles are classified using a single value decomposition approach. The spatial distribution of the magnetic signal shows that profiles of different classes (having different velocities, splitting, asymmetries) are clustered together and form patches, close to the spatial resolution in size. Most of the field is found to be located in intergranular lanes. The statistical properties of the mainly inter-network field sampled by these observations are presented, showing that most of the observed fields are weak with relatively few $\mathrm{kG}$ features. The field strength distribution peaks at $350 \mathrm{G}$ and has a $F W H M$ of $300 \mathrm{G}$. Other parameters, such as profile asymmetries, filling factors and line-of-sight velocities are also determined and discussed.
\end{abstract}

Key words. Sun: photosphere - Sun: magnetic fields - Sun: infrared - polarization

\section{Introduction}

Solar magnetic fields outside sunspots and active regions are believed to exist in the form of a network composed of spatially unresolved flux tubes of $\mathrm{kG}$ field strength (e.g. Frazier \& Stenflo 1972; Stenflo 1973; Solanki 1993; Grossmann-Doerth et al. 1996) and a weaker component located inside supergranular cells (Livingston \& Harvey 1971; Zirin 1985, 1993). The magnetic features composing the latter are known as internetwork fields (IN). Studies of these fields are comparatively scarce due to the severe observational requirements imposed on their detection by their small magnetic fluxes.

Send offprint requests to: E. V. Khomenko,

e-mail: khomenko@mao.kiev.ua

* Based on observations with the German Vacuum Tower Telescope (VTT) operated by the Kiepenheuer-Institut für Sonnenphysik at the Spanish Observatorio del Teide of the Instituto de Astrofísica de Canarias (IAC).

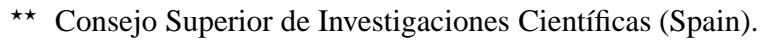

The flux in an inter-network element is an order of magnitude smaller than in a typical network element. The intrinsic field strength in inter-network regions is definitely lower than that in the network, but the typical values determined so far are contradictory. The values of the magnetic field strength in the quiet Sun deduced from observations in visible lines are systematically larger than from infrared lines (e.g. Grossmann-Doerth et al. 1996; Sánchez Almeida \& Lites 2000; Socas-Navarro \& Sánchez Almeida 2002). The first spectral observations in circular polarization in visible lines were performed by Keller et al. (1994), who found evidence that inter-network fields have an intrinsic strength below $\mathrm{kG}$. The upper limit set by these authors for four studied IN elements was $500 \mathrm{G}$ with a probability of $68 \%$ and $1 \mathrm{kG}$ with a probability of $95 \%$.

Observations in the infrared at $1.56 \mu \mathrm{m}$ by Lin (1995), gave a broad distribution of field strengths in the quiet Sun (his analysis included both network and inter-network regions) with a maximum at about $500 \mathrm{G}$ for magnetic elements with flux above $1.5 \times 10^{16} \mathrm{Mx}$ per pixel $\left(10^{15} \mathrm{~cm}^{2}\right)$. This flux value was 
the lower limit on the detectable flux imposed by noise in the observations. By combining the polarization signals from various pixels Solanki et al. (1996) were able to lower the noise in the data of Lin (1995) and thus determined more reliable field strength values, although at the cost of the statistics. For the weakest flux element, the value of the intrinsic field strength obtained was $B=280 \pm 160 \mathrm{G}$. That the field strengths of the IN elements are well below $\mathrm{kG}$ was confirmed also by Meunier et al. (1998) based on a statistical analysis of $1.56 \mu \mathrm{m}$ data.

As was argued recently by Bellot Rubio \& Collados (2003), the determination of the intrinsic field strength in inter-network from visible lines can to a large extent be affected by noise, leading to an overabundance of $\mathrm{kG}$ fields. Thus, the determination of the exact values of the field strength in the inter-network require better statistics and improved (i.e. lower noise) infrared spectropolarimetric data.

On a large scale, inter-network magnetic fields are believed to be distributed more or less uniformly over the solar surface. On a small scale, most of the magnetic field concentrations are located at places where horizontal flows converge. At low fluxes, the sizes and lifetimes of IN fields are closely related with those of granulation (the so-called "granular" magnetic fields observed by Lin \& Rimmele 1999, with a flux detection limit of $5 \times 10^{15} \mathrm{Mx}$ ). The lifetime of the "granular" magnetic fields is of the order of minutes, while the lifetime of the larger flux features is of the order of hours.

Stokes $V$ profiles observed in the quiet Sun have a broad range of asymmetries. For mainly network fields, the average amplitude and area asymmetries are about $15 \%$ and $5 \%$, respectively, suggesting velocity and magnetic field gradients along the line-of-sight (Grossmann-Doerth et al. 1996; Sigwarth et al. 1999). The Stokes $V$ asymmetry of IN fields has been less well studied. It is known that the fraction of "anomalous" profiles (i.e. without the usual double-lobe shape) increases with decreasing magnetic flux. Such anomalous Stokes $V$ profiles are indicative of mixed polarities. From spectropolarimetric data of a quiet Sun region that contained network and IN areas observed in the visible Fe I lines around $\lambda 6302$ ̊, Socas-Navarro \& Sánchez Almeida (2002) found that $35 \%$ of the observed weakest Stokes $V$ signals in the quiet Sun require mixed polarities. While in active regions, mixed polarity profiles are located mainly along polarity inversion lines, in the quiet Sun these profiles also occur in the form of individual patches (e.g. Sigwarth 2001).

Is the IN magnetic field of "turbulent" origin or is it better described by a model based on the flux tube concept? In contrast to the flux tube model that incorporates at least twocomponent atmospheres inside a pixel (non-magnetic and magnetic with the possibility of mixed polarities if more than one magnetic component is allowed), the standard turbulent field model assumes one space-filling single-valued magnetic component with random orientation of the field vector, although a distribution of field strength values can also be incorporated (e.g. Stenflo 1999; Trujillo Bueno 2003). Solanki et al. (1996) provided support for the idea that IN magnetic fields are better described by flux tubes than by turbulent fields. They showed that the dependence of the intrinsic field strength on spatially averaged field strength (a proxy for magnetic flux) is in qualitative agreement with the predictions of models of flux tube formation by the convective collapse mechanism (Parker 1978; Webb \& Roberts 1978; Spruit 1979; Spruit \& Zweibel 1979; Venkatakrishnan 1986). The field strength of weak flux features shows a strong dependence on their flux, while the field strength of the intense features is almost flux independent. The convective collapse mechanism starts to work, with increasing efficiency, as the size and, hence, the flux of a feature increases. In the observations of Lin \& Rimmele (1999), the flux of the features was not strong enough for the convective collapse to take place and only one example of the formation and quick disappearance of a $\mathrm{kG}$ feature was observed. However, as speculated by Lin \& Rimmele (1999), the absence of the formation of $\mathrm{kG}$ fields at low fluxes can be due to the turbulent character of the field, for example, if it is generated by turbulent plasma motions near the solar surface (e.g. Cattaneo 1999). In this case, the dynamics, sizes and lifetimes of the magnetic field will be closely related to that of granulation with a wide distribution of sizes and field strengths biased towards the smallest values.

Further theoretical and observational investigations are needed to answer the question of the nature of inter-network fields. In this paper we present the results of an analysis of spectropolarimetric observations of the full Stokes vector of the Fe I $\lambda 15648 \AA$ and Fe I $\lambda 15653 \AA$ lines recorded with the Tenerife Infrared Polarimeter (TIP) attached to the German VTT in Tenerife. The data combine high polarimetric and Zeeman sensitivity with high spatial resolution, allowing an accurate determination of the properties of IN elements. The detection of IN fields depends strongly on the seeing conditions. We, therefore, compare two observations of very quiet regions near disc centre taken during two independent observing campaigns. This allows us to estimate the effects produced by the different seeing conditions during the two observing days on the properties of the observed magnetic features, e.g. the percentage of mixed polarity profiles and the size of the patches formed by the IN fields. In Sect. 2 we describe the observations and the data analysis procedure. We also attempt to classify the IN Stokes profiles in an objective manner. To this end, as detailed in Sect. 3, we apply a Principal Component Analysis (PCA) to the data. Section 4 focuses on a discussion of the main results and summarizes our conclusions.

\section{Observations and data reduction}

Spectra of the full Stokes vector were recorded during two independent observing runs using the Tenerife Infrared Polarimeter (TIP; see Martínez Pillet et al. 1999) attached to the echelle spectrograph of the $70 \mathrm{~cm}$ German VTT operating at the Spanish Observatorio del Teide (Tenerife) of the Instituto de Astrofísica de Canarias. The first dataset was obtained on July 29th, 2000 and the second on September 5th, 2000. In both cases, a quiet region located at disc centre was scanned, and the presence of plage or network magnetic signals were intentionally avoided to improve the statistics of observed weak IN fields, located inside supergranules. This was done with the help of on-line monitors showing the same region in the $\mathrm{H}_{\alpha}$ 
and Ca II K spectral lines. The network brightness enhancement was clearly distinguishable, especially, in the calcium image, and allowed us to avoid network regions.

Unfortunately, these slit-jaw images were not recorded in a format permitting a quantitative scientific use. Nonetheless, a possible photometric definition of the network areas based on the $\mathrm{Ca}$ II $\mathrm{K}$ brightness would be of little usefulness for other investigations, since the contrast of the images depends on the properties of the particular filter used and its wavelength transmission band, so that no unique criterion can be applied. Indeed, it is an open question to what extent internetwork and network features form distinct families which can be distinguished in a unique and physically sensible manner.

Figure 1 shows the broad-band white light image of the July observations. The excellent seeing conditions during this run is apparent from the good granular shape definition. The image below corresponds to the Ca II $\mathrm{K}$ image of the same region. The black rectangle outlines the scanned area.

The observed spectral range covered the two Fe I lines $15648 \AA(g=3)$ and $15653\left(g_{\text {eff }}=1.53\right)$, with a sampling of $29.1 \mathrm{~m} \AA / \mathrm{px}$. The slit covered $38^{\prime \prime}$ on the Sun, with a sampling of $0.38^{\prime \prime} / \mathrm{px}$. In both campaigns, the Sun was scanned in the direction perpendicular to the slit with steps of $0.38^{\prime \prime}$. The resulting field of view had a size of $20^{\prime \prime} \times 38^{\prime \prime}$ in July, and about $40^{\prime \prime} \times 38^{\prime \prime}$ in September. This last region, due to its larger size, included a small patch of network.

The seeing conditions were very good and stable during the first observing campaign, with a granulation contrast between 5 and $6 \%$ in a continuum window centered at a wavelength of $500 \mathrm{~nm}$. A correlation tracker (Ballesteros et al. 1996) was used to stabilize the image and to make the scan, while keeping the reference image on pure granular images. The reconstructed continuum image from the infrared intensity spectra $(1.56 \mu \mathrm{m})$ had a granular contrast of $2 \%$. During the second run, the seeing conditions were neither so good nor so stable. The visible image contrast was variable and reached maximum values during the scan of about $3 \%$. For this reason, it was not possible to stabilize the image using granular images as references, and the tracking was done on a small nearby pore, outside the observed region. For this dataset, the reconstructed continuum image from the infrared intensity spectra had a granular contrast of $1.4 \%$.

The data acquisition system operated at a rate of 8 frames s ${ }^{-1}$, thus allowing the full Stokes vector to be recorded at every pixel along the slit and at all observed wavelengths every $0.5 \mathrm{~s}$, approximately. To increase the signal to noise ratio, 120 sets of four images were added up on-line in July, and 100 in September (corresponding to total integration times of 60 and $50 \mathrm{~s}$, respectively, at each position of the slit). After this integration, the noise in the Stokes $Q, U$, and $V$ spectral images was about $2-3 \times 10^{-4}$ in units of the continuum intensity. The total observing time needed to scan the two regions was about 50 and $85 \mathrm{~min}$, respectively. Some examples of polarization maps, spectral images and individual profiles taken from the July dataset can be found in Collados (2001).

Most of the crosstalk between the Stokes parameters induced by the presence of oblique mirrors in the light path inside the telescope can be removed from the data by the use of
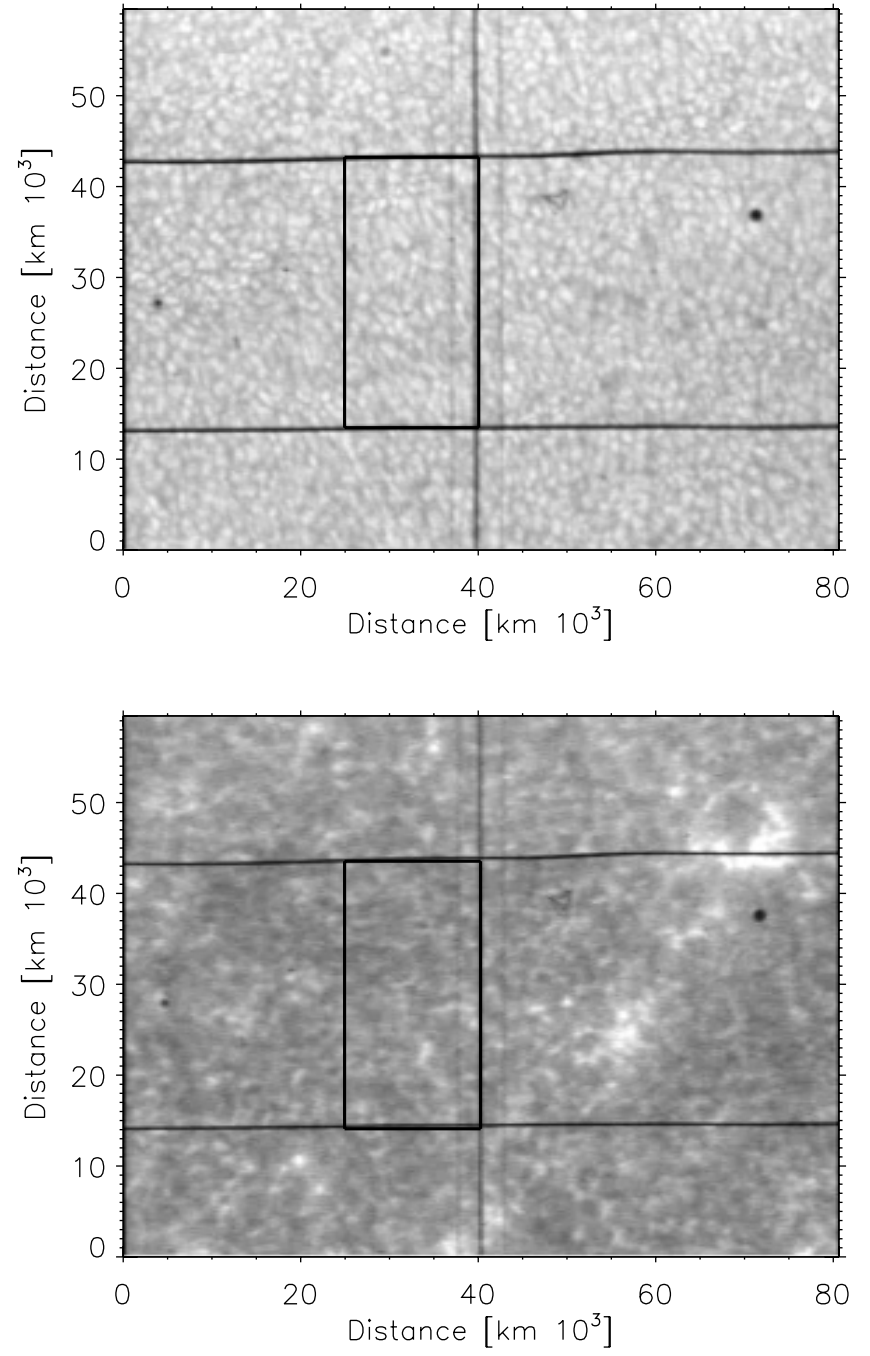

Fig. 1. Slit-jaw images of the scanned area during the July observations. The black rectangle in both images delineates the scanned area. Top: while-light broad-band image. The excellent seeing conditions during this observing run is apparent from the sharpness of the granulation. Bottom: Ca II $\mathrm{K}$ image. The absence of brightness at this wavelength in the scanned rectangle suggests that it is representative of the inter-network.

calibration optics located appropriately in the beam (Collados 1999; Bellot Rubio et al. 2000; del Toro Iniesta et al. 2001; Schlichenmaier \& Collados 2002). The coelostat configuration of the telescope could not be calibrated, however, and a theoretical model for it, using standard aluminium refractive indices, was used (see Collados 1999). The residual crosstalk from Stokes $I$ to Stokes $Q, U$, and $V$ was removed by forcing the continuum of these to zero. After the experience obtained with the analysis of sunspot spectra (see the corrections applied by Schlichenmaier \& Collados 2002), we are aware that a residual crosstalk between Stokes $Q, U$, and $V$ of a few percent may still remain in the data. In addition, the variable seeing conditions during September might have introduced some additional seeing-induced crosstalk between $Q, U$ and $V$ which is difficult to evaluate, but which is certainly not larger than the residual instrumental polarization, as the visual inspection of the 
polarization spectra shows. However, the seeing-induced crosstalk is of random nature, rendering its correction difficult.

An absolute wavelength calibration was not possible due to the absence of telluric spectral lines in the observed spectral window. An approximate calibration was done by selecting all those pixels where the magnetic signal was undetected, i.e. below the noise. The wavelength position of the intensity minimum in the $15648 \AA$ line was then calculated at each spatial point and its average value was defined as the zero velocity wavelength. This value is less affected by the standard convective blueshift than a granulation-averaged intensity profile, because to first order the granular intensity-velocity correlation does not influence the averaged line minimum, as long as the granulation is resolved. However, a residual blueshift of unknown magnitude remains, produced by the unresolved smallscale granular brightness and velocity structure (see Sect. 3.7).

\section{Results}

The amplitudes of the polarization signals in our data are very weak, which is expected for observations of IN fields. The Stokes $V$ amplitudes vary from the noise level up to 0.01 (in units of $I_{\mathrm{c}}$ ). The amplitudes of the $Q$ and $U$ profiles are lower; the maximum values achieved are about 0.004 . In our analysis we used only those Stokes $Q, U$ and $V$ profiles with amplitudes above a threshold level of $10^{-3}$. This threshold value was a compromise between noisy profiles and weak, but interesting signals. This criterion was satisfied in almost $50 \%$ of the pixels for Stokes $V$, and in $20 \%$ for Stokes $Q$ and/or $U$.

\subsection{Classification of Stokes profiles}

All the profiles selected in the way described above were classified using a Principal Component Analysis approach (PCA; see, e.g., Rees et al. 2000) similar to that applied by Sánchez Almeida \& Lites (2000) for the classification of spectropolarimetric observations of a quiet-Sun region using the Fe I lines around $\lambda 6302 \AA$. This PCA strategy allows the number of parameters needed to describe a line profile to be reduced. According to it, every profile $S\left(\lambda_{j}\right), j=1, . ., N_{\lambda}$ (where $N_{\lambda}$ is the number of sampled wavelengths) can be represented by a linear combination of a set of eigenvectors $\boldsymbol{e}_{i}\left(\lambda_{j}\right)$, $(i=1, \ldots, n)$ :

$\boldsymbol{S}\left(\lambda_{j}\right)=\sum_{i=1}^{n} c_{i} \boldsymbol{e}_{i}\left(\lambda_{j}\right)$,

with the appropriate constant coefficients. The system of eigenvectors is obtained from a dataset of example profiles using a singular value decomposition (SVD) method (for details see Rees et al. 2000; Socas-Navarro et al. 2001) and forms an orthonormal vector base, i.e.,

$$
\sum_{j=1}^{N_{\lambda}} \boldsymbol{e}_{i}\left(\lambda_{j}\right) \boldsymbol{e}_{k}\left(\lambda_{j}\right)=\delta_{i k}
$$

The SVD method provides directly such a base, composed of as many vectors $n$ as the number of spectra in the dataset. In practice, it turns out that most of these vectors do not carry information on the spectral line profiles, and are only needed to reproduce the particular noise pattern of the example profiles. Therefore, if the dataset of the example profiles is properly selected, the series expansion (1) can be truncated after the first few terms and only a small number $n$ of the coefficients $c_{i}$ is required to characterize each profile. The coefficient vector $c$ corresponding to a given observed profile $S_{0}(\lambda)$ is obtained from:

$c=e S_{0}$.

This relationship can be directly derived from Eqs. (1) and (2). $\boldsymbol{S}_{0}$ is a column $N_{\lambda}$-dimensional observed profile, $\boldsymbol{c}$ a column $n$-dimensional vector with the coefficients, $n$ is now the number of terms retained in the expansion (1), and $\boldsymbol{e}$ is a matrix whose rows are the retained eigenvectors. It turns out that, generally, many observed line profiles exhibit similar characteristics, so that a few eigenvalues associated with the eigenvectors are much larger than the rest of eigenvalues. This allows the observed profiles to be classified according to their spectral shape, or, in other words, according to which of the eigenvectors contributes the most (i.e., with the largest coefficients) to reproduce a given profile.

We organized the coefficients $c$ describing each of the profiles into a given number of classes using a cluster analysis approach. According to this approach a predefined number of cluster centres (classes) was calculated based on the values of the variables in the data (coefficients $c$ in our case). Each profile is represented by a point in the $n$-dimensional space formed by eigenvectors $\boldsymbol{e}$. A profile was ascribed to a class under the condition that the distance from this point to that particular cluster centre was smaller than to the other cluster centres. After the classification, a typical profile $\boldsymbol{S}_{\text {class }}$ (hereinafter named class profiles) was computed for every cluster or class as follows:

$\boldsymbol{S}_{\text {class }}=\boldsymbol{e}^{T} \overline{\boldsymbol{c}}$

where $\bar{c}$ is the average coefficient of all the profiles belonging to the same class.

We have carried an analysis for Stokes $V$, on the one hand, and for Stokes $Q$ and $U$, on the other. The number of cluster centres found to be sufficient was 8 for $V$ and 5 for $Q$ and $U$. The profiles were normalized (to their corresponding maximum absolute value) and signed positive before the classification (positive blue lobe for the circular polarization profiles, and positive central lobe for the linear ones).

The results of the PCA analysis of the real dataset depend on the choice of the subset used as example profiles for the computation of the eigenvectors. This choice of profiles has to be made in such a way that the initial dataset represents a good statistical description of all the possible profiles present in the full dataset (Socas-Navarro et al. 2001). The construction of such a dataset can be a problem, since the distribution of the profile shapes is a priori unknown.

We used a subset of the observed profiles to build an example dataset. In this case, the $S / N$-ratio plays a critical role in determining the set of eigenfunctions. According to the discussion by Socas-Navarro et al. (2001), the first eigenvectors $\boldsymbol{e}$ carry most of the information about the profiles and are weakly sensitive to noise. Accordingly, by truncating the series (1) at sufficiently small $i$, the influence of the noise should be minimized. However, our tests with artificial profiles showed that 
this is true only if the $S / N$-ratio of the data is sufficiently large. This is a particularly critical requirement when studying IN fields, which only give a weak signal. We found that the classification is stable when the ratio between the amplitude of the profiles selected as examples and the noise level is larger than 10. Thus, low-amplitude profiles should not be employed to build the database in order to ensure a stable classification. Such a cutoff introduces a bias into the classification, however, since the number of irregular profiles in our observations increases with decreasing amplitude, in accordance with the behaviour found by Sánchez Almeida \& Lites (2000). It means that if we construct the initial database only from large amplitude profiles, examples of very asymmetric profiles (e.g. ones having 3 or more lobes) will be missing, and the decomposition of such profiles may not be appropriate. To find a compromise between these two opposite demands, we constructed the initial dataset of 500 profiles randomly selected from the first 5000 sorted by decreasing amplitude.

Since the statistical description of the full dataset is unknown, different selections of these 500 profiles can result in slightly different classifications. We found that the shapes of the resulting $\boldsymbol{S}_{\text {class }}$ fluctuate, leading to changes in the number of profiles belonging to each class. To find the magnitude of the statistical error produced by these fluctuations, we repeated the classification procedure 80 times, taking randomly in each realization 500 different example profiles, and obtained 80 possible sets of $\boldsymbol{S}_{\text {class }}$ profiles. Then, we found the best correspondence between the sets of $\boldsymbol{S}_{\text {class }}$ from the different realizations. For that, one of the sets $\boldsymbol{S}_{\text {class }}(i, j)$ (where $i=1, \ldots, n$ is the class number, and $j=1, \ldots, 80$ is the realization number) was chosen as a reference and correlation was found between this set and, successively, the others by varying $i$ for each fixed $j$. Then, the average of 80 profiles with the best correlation (separately for each class) was defined as a new class profile. The same observed profile can be ascribed to different classes due to variations of $\boldsymbol{S}_{\text {class }}$ from one realization to the next. Finally, we took the criterion that a profile belongs to the class it has been ascribed the maximum number of times during the 80 realizations. The standard deviations in the number of profiles belonging to a class during the 80 realizations were taken as a measure of the statistical error.

\subsection{Stokes $V$}

Figure 2 shows the 8 classes of Stokes $V$ profiles $\left(\boldsymbol{S}_{\text {class }}\right)$ produced by the IN regions of the quiet Sun. The classes were obtained from the analysis of the July and September datasets together in order to make the classification statistically more reliable. The noise level and the seeing conditions were different in July and September. However, our tests with a separate classification of the two datasets returned very similar classes. The various types of Stokes $V$ profiles shown in Fig. 2 are sorted by decreasing percentage of observed profiles belonging to each of them. These numbers are different in the July and September datasets, and the corresponding values are given in Fig. 2 together with the standard deviations.
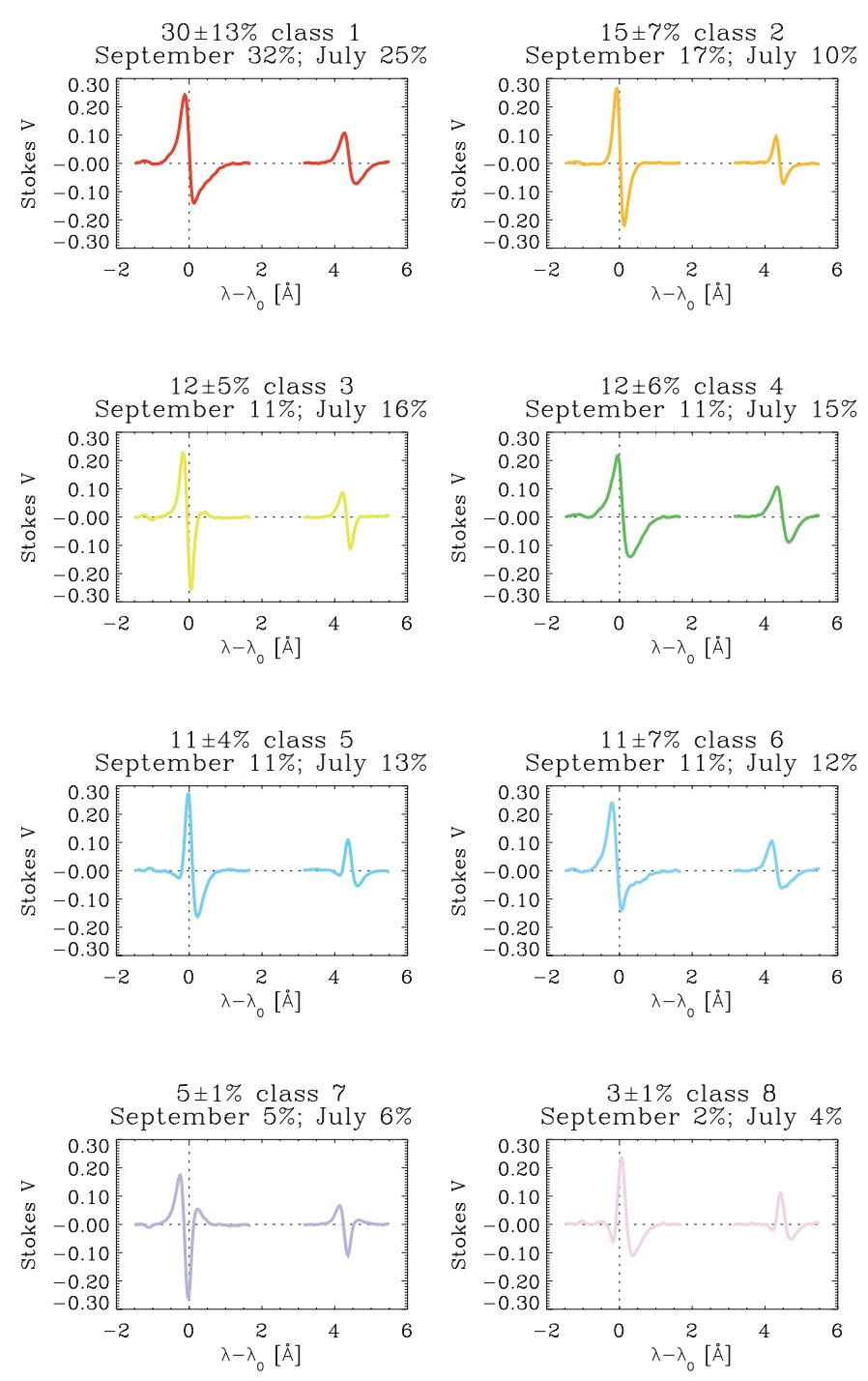

Fig. 2. Stokes $V$ class profiles $\boldsymbol{S}_{\text {class }}$ resulting from a PCA classification. Vertical dotted lines indicates the average wavelength of the $\lambda 15648 \AA$ line in the nearly field-free part of the quiet Sun (see Sect. 2). Horizontal dotted lines indicate the continuum or zero level. The two spectral lines were classified simultaneously. The values at the vertical axis are arbitrary. The number of the profiles belonging to each class is given in percent at the top of each panel. The values are given for the July and September data separately and for both datasets together. The variation in the number of profiles is a standard deviation from the mean value (see text for details). The classes are sorted out according to the number of observed profiles assigned to them.

The various classes can differ from each other in the amount of Zeeman splitting (e.g. classes 1 and 2), the line shift (e.g. classes 1 and 4), the asymmetry (e.g. classes 2 and 7), higher order details of the line shape (e.g. classes 1 and 6) and any combination of the above, but not the $V$ amplitude or sign. Some parameters of the class profiles are given in Table 1. The values of the magnetic field strength, Stokes $V$ zero-crossing wavelength and amplitude and area asymmetries listed in the table were determined directly from the class profiles $\boldsymbol{S}_{\text {class }}$ shown in Fig. 2 (see the discussion below for the definition of these values). The Stokes $V$ amplitudes given in the Table 1 were 
Table 1. Parameters of Stokes $V$ class profiles. The first column represents the class number (see text). The second column is the number of the profiles belonging to each class. $B$ and $v_{z c}$ are, respectively, their average intrinsic magnetic field strength and Stokes $V$ zero-crossing wavelength (positive means downflow). The last three columns are the average $V$ amplitude and the average asymmetries of Stokes $V$ amplitude and area (see Sect. 3.8).

\begin{tabular}{ccccccc}
\hline \hline Class & $\begin{array}{c}N \text { of profiles } \\
{[\%]}\end{array}$ & $\begin{array}{c}B \\
{[\mathrm{G}]}\end{array}$ & $\begin{array}{c}v_{\mathrm{zc}} \\
{\left[\mathrm{km} \mathrm{s}^{-1}\right]}\end{array}$ & $\begin{array}{c}\text { Amplitude } \\
{\left[10^{-3} I_{\mathrm{c}}\right]}\end{array}$ & $\begin{array}{c}\text { Ampl. asym. } \\
{[\%]}\end{array}$ & $\begin{array}{c}\text { Area asym. } \\
{[\%]}\end{array}$ \\
\hline 1 & $30 \pm 13$ & 470 & 0.2 & 1.98 & 27 & 6 \\
2 & $15 \pm 7$ & 300 & 0.3 & 2.08 & 9 & 2 \\
3 & $12 \pm 5$ & 280 & -1.3 & 1.95 & -7 & 13 \\
4 & $12 \pm 6$ & 610 & 1.7 & 1.65 & 21 & 1 \\
5 & $11 \pm 4$ & 370 & 1.6 & 1.49 & 26 & -4 \\
6 & $11 \pm 7$ & 490 & -1.2 & 2.00 & 27 & 12 \\
7 & $5 \pm 1$ & 370 & -3.0 & 1.84 & - & - \\
8 & $3 \pm 1$ & 320 & 3.7 & 1.37 & - & - \\
\hline
\end{tabular}

determined from the observed $V$ profiles by averaging over all the values for the corresponding class. The profiles of classes number 3, 5, 7 and 8 have a more or less well-defined 3-lobe shape and arise from pixels where mixed polarities with different velocities are unresolved (e.g. Rüedi et al. 1992). They represent about $30 \%$ of all profiles in our data. Note that class 3 provides the largest contribution to these multi-component profiles, and for this class the contribution from a second magnetic component is very small (which is also the case for the class 5). In these cases we have therefore still employed the standard definition of area and amplitude asymmetry (see Sect. 3.8). For classes 7 and 8 the 3-lobe structure is very clear and we have refrained from determining these parameters. The anomalous Stokes $V$ profiles are more frequent in July (39\% vs. $29 \%$ in September). The profiles of class number 6 are probably produced by two magnetic components of the same polarity but having different velocities and field strengths (e.g., Rüedi et al. 1992). Very likely, the coexistence of a weak and a strong $\mathrm{kG}$ component needs to be invoked for their explanation, as suggested by the illustrative synthetic IR profiles shown by SocasNavarro \& Sánchez Almeida (2003), which were obtained by adding the contributions of both sub-kG and kG fields. The profiles of classes number 1 and 4 have a similar shape, but the profiles of class number 4 are more shifted to the red (downflow) and have a larger splitting between the lobes (see Table 1). Class number 2 contains profiles coming from unipolar regions with a very weak field strength. Despite the small splitting, the amplitudes of these profiles are quite large in comparison to the rest. Typical profiles of all classes, except for classes number 2 and 3 , are very asymmetric (see Table 1 ).

The spatial distribution of the Stokes $V$ profiles belonging to different classes is presented in Fig. 3. It can be seen that the different classes of Stokes $V$ profiles change rapidly over small regions and that profiles of the same class are not randomly located, but form patches.

One can gain an idea of the spatial structuring of the field from the transformations of the patches of different classes from one to another. The profiles of classes 1, 4 and 6 have the largest splitting (470 $\mathrm{G}$ for class 1 and $610 \mathrm{G}$ for class 4 , see Table 1). For class 6 the splitting listed in Table 1 probably lies between the splitting of the 2 components forming such a profile. In the following we discuss classes 1 and 4, which likely can be described by a dominant single component. The profiles of class 4 are coloured green and are the most redshifted of all classes, while the profiles of class 1 (red) are on average almost unshifted $\left(v_{\mathrm{zc}}\right.$ for class 8 given in Table 1 is larger than that of class 4 , but the shift of the zero-crossing in this case is likely produced by the presence of at least two unresolved components in the pixel). The patches formed by the profiles of these two classes have the largest sizes and often touch each other. In the September data, most of the profiles of classes 1 and 4 are of the same polarity. Since the network can be unipolar over large distances this can indicate that a part of such profiles may originate from a piece of network present in the scan (upper right corner of the lower panel in Fig. 3). The field strengths are the largest in the mentioned region and reach $\mathrm{kG}$ values. Examples of the profiles of these classes in the July data are patches located at position $X=20$ and $Y \approx 0$, at the $X$ position between 15 and 20 and $Y \approx 4$; at $X$ from 2 to 5 and $Y \approx 7$, and others. In the July data, these patches are not grouped and have different polarities.

The patches of the profiles of other classes are smaller. There are some unipolar regions with profiles of class 2 in the September data (coloured orange) which are almost absent in July. These areas are often mixed with patches of 3-lobe profiles of class number 5 . The mixed polarity profiles of class 5 form aureoles around the locations of the profiles of class 2 . Examples are the patches between $X$ coordinate 10 and 13 and $Y$ coordinate from 23 up to 30, $X$ between 10 and 25 and $Y$ between 0 and 5 in the September map. The patches of such profiles are often located in between opposite polarity areas. However, isolated patches of 3-lobe profiles can be found in the July map (but are almost absent in September). The profiles of class number 6 (dark blue) are often located between profiles with large and small Zeeman splitting, which is not surprising given that profiles of this class are probably composites of two profiles with different splittings.

The class profiles represent the statistical distribution of all the possible profiles produced in the inter-network region. Summarizing, the observed regions in July and September 

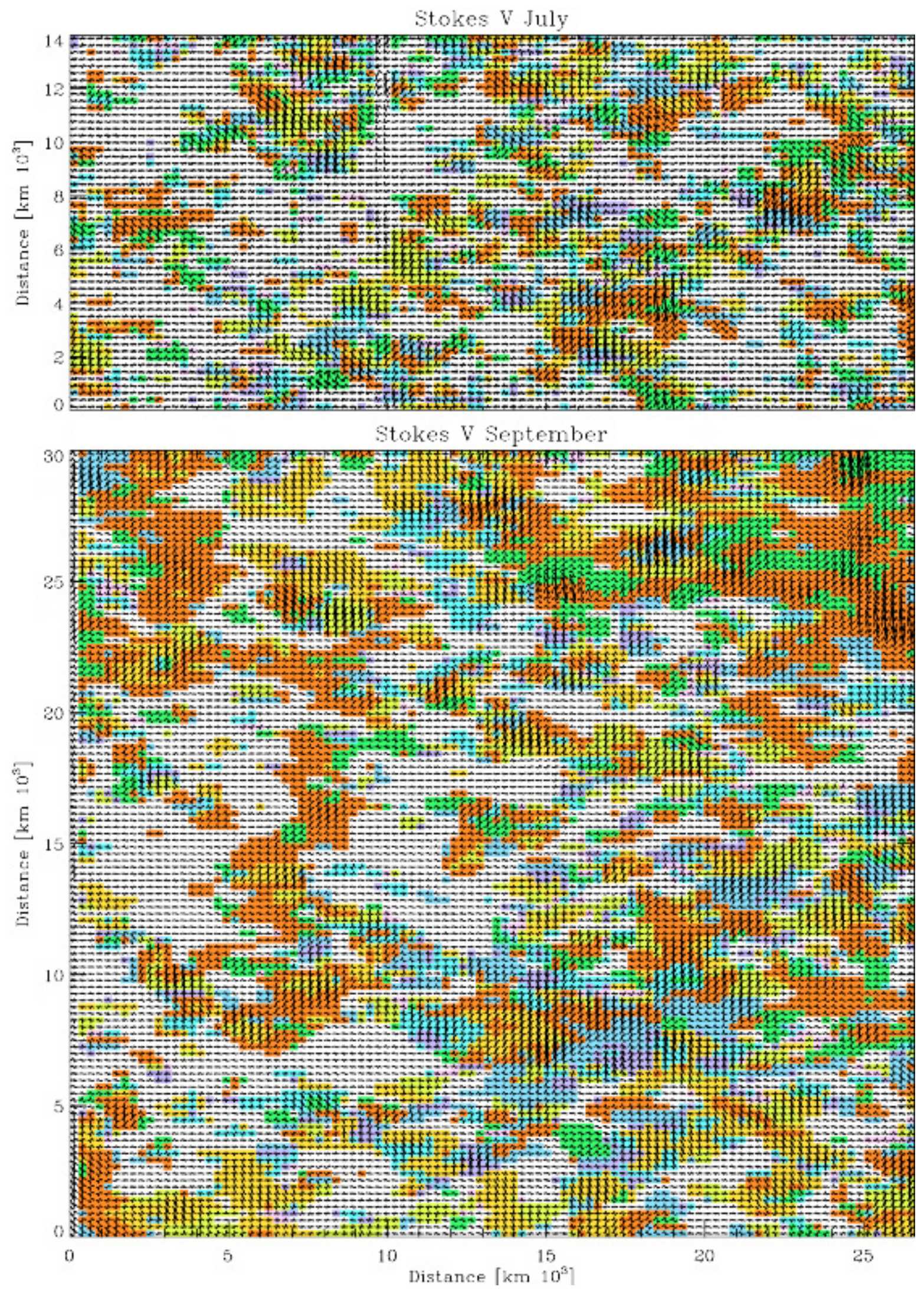

Fig. 3. Stokes $V$ maps of the inter-network regions. Upper panel: July data; lower panel: September data. The horizontal direction corresponds to the direction of the slit, the vertical to the scan direction. Each pixel is represented by a coloured square with the corresponding Fe I $15648 \AA$ Stokes $V$ profile drawn inside. The different colours represent the various classes of profiles. The colour coding is the same as in Fig. 2 . The two pixel columns with noisy profiles in the July map are due to a bad flatfield correction. 
contain a wide variety of profile shapes. The classification shows that $57 \%$ of the observed pixels with signal above the threshold contain fields that can be described by a single dominant magnetic component, corresponding to the profiles of the classes 1, 2 and 4. Areas with mixed polarities and different velocities occupy $32 \%$ of the area in total (classes $3,5,7,8$ ), while in $10 \%$ of the magnetic features several components with the same polarity, but different field strengths and velocities are present (profiles of the class 6). The statistical distribution of the profiles of different classes is similar in July and September, except for the fact that in September more homogeneous and less mixed polarity fields were observed. In general, the spatial scale on which the field changes (in the sense that it requires different classes to describe it) is about $1^{\prime \prime}$. This value is close to the granular scale and to the spatial resolution (see below).

The classes of Stokes $V$ profiles for the infrared lines are different from those found in the visible (see Fig. 4 of Sánchez Almeida \& Lites 2000). The reason for this can reside in the different Zeeman sensitivity of visible and IR lines (see Harvey \& Hall 1975; Solanki et al. 1992; Solanki 1994; Stenflo 1994; Socas-Navarro \& Sánchez Almeida 2003, and references therein). The fraction of Stokes $V$ profiles having an irregular shape is much larger in the infrared. In any case, Socas-Navarro \& Sánchez Almeida (2002) found that the fraction of irregular profiles in their data increases with decreasing Stokes $V$ amplitude and about $35 \%$ of the weakest signals require mixed polarities.

The main difference between the July and September data is a larger size of patches in the latter dataset. It is on average about $0.95^{\prime \prime}$ in the July data and $1.25^{\prime \prime}$ in the September data. There can be several reasons for this. On the one hand, the observed fields could be intrinsically different due to the presence of network in the observed area in September (see Sect. 3.6).

On the other hand, the difference in the data can be caused by a non-solar phenomenon. The seeing conditions during the observations of both days seem to be an important parameter that can influence the size of the patches. Seeing causes a greater spatial smearing, so that a signal at a pixel becomes a combination of the true solar signal at this point and a fraction of the signals at the nearby points. If the signal at some pixel is strong it would cause all the nearby pixels to have a similar type of profile. Under good seeing conditions the contamination from the nearby pixels is smaller and more details can be resolved. This makes the areas with a similar signal smaller. The rms of the infrared continuum intensity in July was $2 \%$, but only $1.4 \%$ in September. Also, the seeing conditions were more stable during the observations in July. The estimated size of the smallest intensity structures resolved in our data from the spatial power spectra of the continuum intensity image was about $1^{\prime \prime}$ in July and about $1.4^{\prime \prime}$ in September. This is an upper limit for the spatial resolution. Hence, the average sizes of patches of the profile classes on the maps are close to our spatial resolution estimates.

Another effect produced by seeing can be a decrease in the number of the observed 3-lobe profiles or those with irregular shape. The September dataset contains more unipolar fields (profiles of the classes 1,2, 4 have mainly the same polarity). Thus, naturally, the number of the mixed-polarity profiles in such region should be smaller. However, given that the signal in September was generally stronger, the spatial smearing would reduce the number of mixed-polarity (lower amplitude) profiles even more. The weakness of the signal in July in contrast to the more unipolar and stronger signals in September together with greater spatial smearing during the latter observations can lead to the observed difference in the number of 3-lobe profiles and in the size of the patches.

We conclude that seeing is an important reason for the difference in our two analyzed datasets. With increasing spatial resolution the field shows more fine-scale structure and fluctuations. The areas formed by the profiles of the same class become smaller and the field changes occur at shorter spatial scales. This suggests that the characteristic scale of the field in the inter-network is smaller than the current resolution of highsensitivity ground-based observations.

\subsection{Stokes $Q$ and $U$}

Most of the previous investigations of inter-network fields were based only upon the analysis of Stokes $V$. There are only a few investigations of quiet Sun magnetic fields based on the full Stokes vector (e.g. Lites et al. 1996; Lites 2002), which is not surprising given the fact that for intrinsically weak fields the amplitudes of Stokes $Q$ and $U$ of lines in the visible are much smaller than that of $V$, even for a field inclined by $45^{\circ}$ to the line of sight. This situation is improved when a very Zeeman sensitive line, such as Fe I $15648 \AA$ is employed (Solanki et al. 1992), so that we expect our data to contain more reliable information on Stokes $Q$ and $U$ than observations in the visible. Even then, only $20 \%$ of the Stokes $Q$ and $U$ profiles in our data have amplitudes above the threshold level. The selected linear polarization profiles were subjected to a PCA analysis. We have carried out the analysis of Stokes $Q$ and $U$ profiles together since we find no systematic difference between the $Q$ and $U$ profiles. Due to the smaller amplitudes of these profiles relative to Stokes $V$, the results of the PCA analysis are more affected by noise and are more uncertain. Also, the number of the statistically analyzed profiles is considerably smaller. However, we were still able to distinguish 5 classes of the linear polarization profiles. These classes are presented in Fig. 4.

Just like Stokes $V$, the typical profiles of the $Q$ and $U$ classes differ in asymmetry, width and wavelength shift. Interestingly, the amplitude of the red $\sigma$-lobe is often more intense than the blue one. In the case of the $15653 \AA$ line this lobe can even be absent (e.g. class 1). A significant fraction of the Stokes $Q$ and $U$ profiles (24\%) have shapes similar to Stokes $V$. Those are profiles of class 4 and, possibly, class 5 . Now, Stokes $Q$ and $U$ change sign for drastic changes in the azimuth $(\chi)$. The profiles belonging to class 5 have low amplitudes and a part of them are very close to the noise level. This suggests that these irregular profiles are formed by cancellation of $Q$ or $U$ profiles of opposite sign. The profiles of the class 4 are more frequent in the September data. There is a possibility that a part of these profiles appear due to, for example, seeinginduced crosstalk from $V$ into $Q$ and $U$ (Collados 1999). Since the amplitudes of Stokes $V$ were larger in September and the 

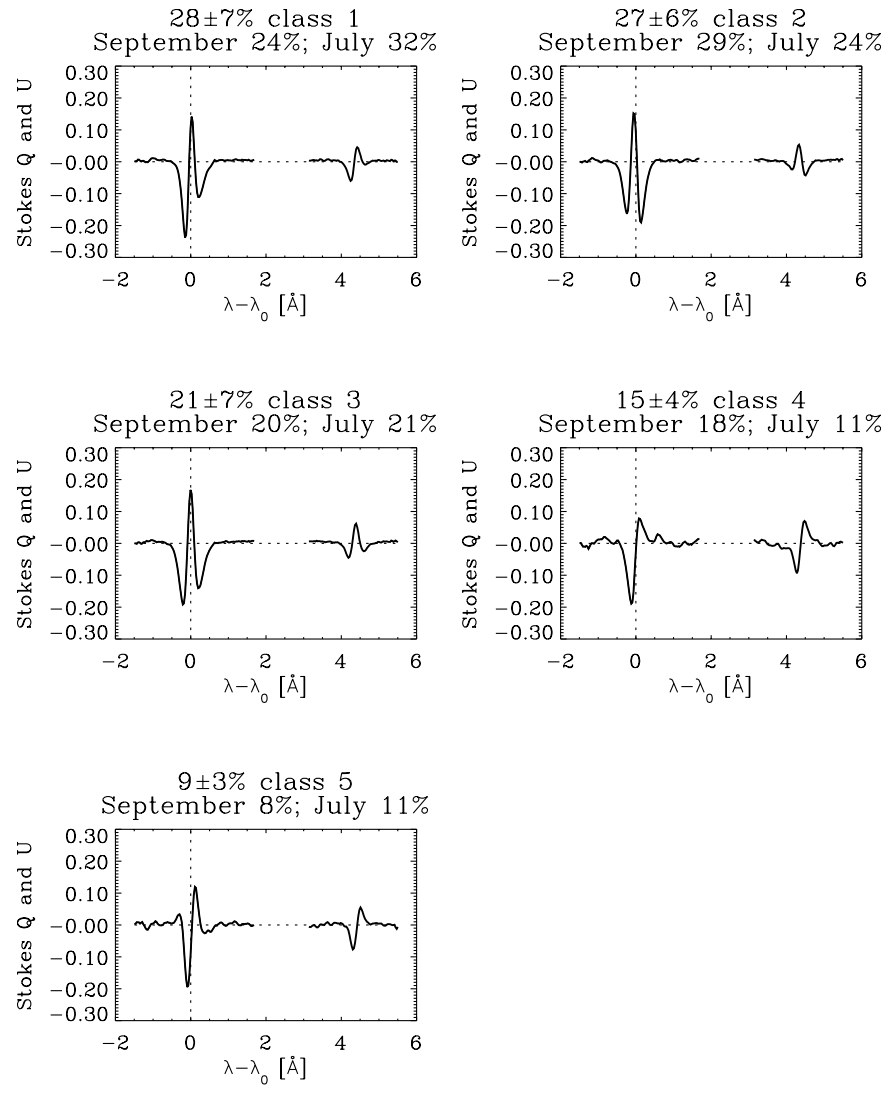

Fig. 4. Stokes $Q$ and $U$ class profiles $\boldsymbol{S}_{\text {class }}$ resulting from a PCA classification. All the notations are the same as in Fig. 2.

seeing conditions were worse, the crosstalk could be larger for this dataset. In case these $Q$ and $U$ profiles are of solar origin, the amplitudes of the two Fe I $\lambda 15648 \AA$ and Fe I $\lambda 15653 \AA$ lines in linear polarization should scale as the square of their Landé factors. Indeed, the amplitude ratio for the profiles of the classes $1-3$ is close to this value $(\approx 4)$. But, it is lower (approximately 2.5-3) for the profiles of class 4 .

While most of the Stokes $V$ profiles are shifted to the red, the Stokes $Q$ and $U$ class profiles do not show this tendency. They are either not shifted with respect to the zero wavelength position or they are slightly shifted to the blue. The difference between the Stokes $V$ zero-crossing wavelength $v_{\text {zc }}$ and the Stokes $Q$ or $U \pi$-component position is about $0.5 \mathrm{~km} \mathrm{~s}^{-1}$.

The linear polarization profiles of different classes also form patches. The size of the patches in July and September data does not differ much in this case. If seeing affects the size of patches in Stokes $V$, it should affect it to a similar extent in the case of other Stokes parameters. However, the patches of significant Stokes $Q$ and $U$ profiles are relatively isolated and the amplitudes of Stokes $Q$ and $U$ are smaller than those of $V$. Hence, the contamination of the neighboring pixels caused by seeing effects may not be sufficient to produce a significant difference in the patch size (i.e. the signal in the neighboring pixels still remains below the noise even after smearing). The patches of linear polarization profiles are located everywhere across the region. Sometimes the signal in Stokes $V$ is absent while it is present in Stokes $Q$ and $U$, meaning that the field lines are relatively horizontal. The fraction of the significant $Q$ and $U$ profiles which are not accompanied by $V$ is about $8 \%$. Horizontal fields seem to be unrelated to regions of changing polarity (see Sect. 3.4). The profiles of the $V$-like shape of classes 4 and 5 also form patches.

\subsection{Magnetic field strength}

In Sects. 3.4-3.8 we discuss the magnetic field strength (determined from the Zeeman splitting), velocities, amplitudes and other line parameters. For their determination we used only profiles of regular shape with a well-defined zero-crossing and two lobes (Stokes $V$ profiles of classes 1, 2, 4, 6 from Fig. 2) and 3-lobe profiles for Stokes $Q$ and $U$ (classes 1, 2, 3 from Fig. 4). About $30 \%$ of the significant Stokes $V$ signals and $24 \%$ of Stokes $Q$ and $U$ did not meet this criterion.

In order to determine the intrinsic field strength $B$, we applied a procedure similar to that of Lin (1995) and Rabin (1992). Each observed $V$ profile was represented by a sum of two Gaussians. We have fitted both spectral lines and have 8 free parameters of the fit. We assume that the velocity field is the same at the level of formation of both lines and that the area and amplitude asymmetries of both lines are the same. Thus, the 8 free parameters used were the amplitudes of the Gaussians and the width of both $\sigma$-components of the $g=3$ line, the Gaussian amplitude and width of the first $\sigma$-component of the $g_{\text {eff }}=1.53$ line, and the positions of the Gaussian $\sigma$-components of the $g=3$ line. The reason for the Gaussian fit approach is that despite its large Zeeman sensitivity, even the $g=3,15648 \AA$ line is not completely split for the weakest fields in the observed region. In the case of Stokes $Q$ and $U$ profiles, only the $g=3$ line was fitted, since $Q$ and $U$ profiles of the $g_{\mathrm{eff}}=1.53$ line are very much smaller and often strongly affected by noise. Three Gaussians ( 9 free parameters) were used. For Stokes $Q$ and $U$, the optically thin assumption underlying this approach is far more critical than for Stokes $V$, due to the presence of the $\pi$ component at line centre. However, the $15648 \AA$ line is sufficiently weak in the quiet Sun for it to be reasonable (Solanki et al. 1987). The splitting was defined as the difference in the wavelength positions of the Gaussians of the two $\sigma$-components and transformed into $\mathrm{G}$. A good fit was achieved for most of the profiles except for the very asymmetric ones.

In practice, the sensitivity to Zeeman splitting of the fit is limited by the width of the spectral lines. We tested the line fitting algorithm on a sequence of synthesized $V$ profiles with the same width as the observed $I$ profiles but different splitting, and found that it is able to retrieve the original splitting

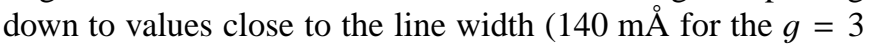
line). Thus, the minimum detectable field strength with our fitting procedure of Stokes $V$ is about 200 G. For smaller splittings the fitting procedure does not have adequate information to separate the effect of the amplitudes of the Gaussians and their separation. Thus, the uncertainty of the field strength determination increases toward smaller splittings (see the discussion by Lin 1995) and the results should be taken as estimates. In the presence of noise, asymmetries, etc. a more conservative estimate of $300 \mathrm{G}$ for the lowest reliably determined 

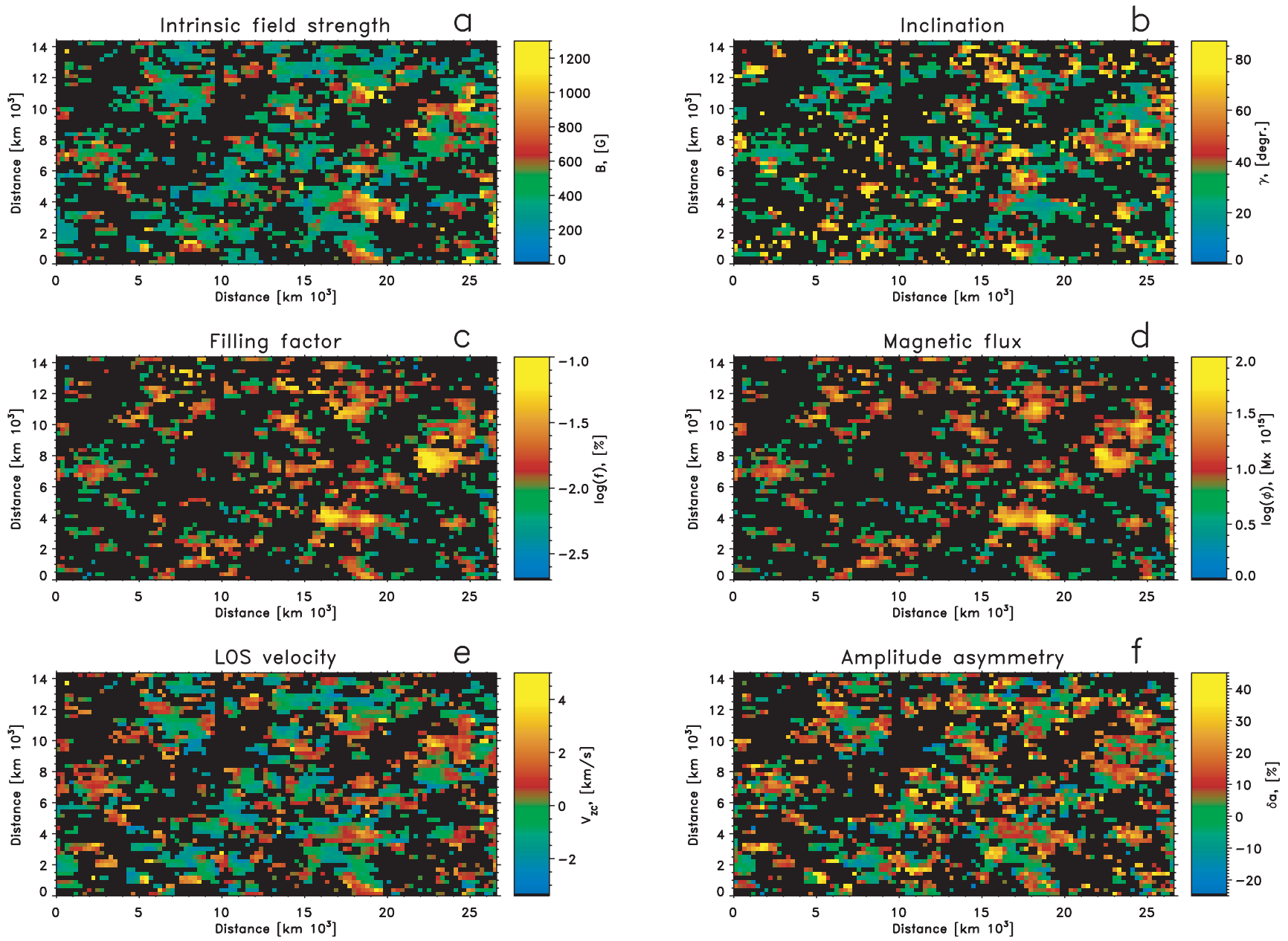

Fig. 5. Distribution of various parameters over the inter-network region (July data). Panels (from the left to the right, from the top to the bottom) are intrinsic unsigned magnetic field strength, inclination of the magnetic vector, logarithm of the filling factor, logarithm of magnetic flux, Stokes $V$ zero-crossing velocity, $V$ amplitude asymmetry. The values of each parameter are indicated by the corresponding colour bars. Pixels coloured black either harbor signal below the threshold or profiles of 3-lobe classes for Stokes $V$.

intrinsic field strength may be more appropriate. Preliminary results from the inversion of the July dataset using the SIR inversion code (Ruiz Cobo \& del Toro Iniesta 1992) give a shape of the distribution of the field strength similar to the one obtained from the Gaussian fit algorithm (see below), but the values of the field strength are lower (Collados 2001).

In the case of Stokes $Q$ and $U$, the $\sigma$-components must separate not just from each other, as in $V$, but rather from the $\pi$ component. Consequently, the line fitting algorithm in this case is limited to a somewhat larger field strength than for Stokes $V$. Our tests with synthesized Stokes $Q$ and $U$ profiles demonstrated that the minimum detectable field strength is about $350 \mathrm{G}$. However, a more conservative estimate of 450-500 G should be applied in the case of real profiles.

Figure 5a shows a map of the intrinsic magnetic field strength in the observed region in July. The field strength in the patches is mostly weak, of about 300-600 G. There are also several patches with stronger field strengths up to $\mathrm{kG}$ values. In such cases, the intrinsic field strength inside a patch is usually higher at the centre and is weaker in the external part. At the same time, the patches of the weaker fields have a more or less homogeneous field distribution. Comparing with the distribution of magnetic field strength in the quiet Sun obtained from the $6301 \AA$ and $6302 \AA$ Fe I lines (Socas-Navarro \& Sánchez Almeida 2002) it can be seen that IR lines show much more polarization signals in regions far away from the network.

Figure 6 shows histograms of the intrinsic magnetic field strength derived from the splitting of the Stokes $Q, U$ and $V$ profiles in our data. The histogram of the Stokes $V$ splitting (left panel of Fig. 6) peaks at $350 \mathrm{G}$, but has a long tail to higher values, well into the $\mathrm{kG}$ range. About $4 \%$ of all fitted profiles give $B \geq 1 \mathrm{kG}$. The field strength values are in agreement with those given by Lin (1995) and Lin \& Rimmele (1999). This confirms that indeed the two scans managed to avoid the network to a large extent, so that almost all the signal seen here is due to IN fields. The histogram drops rapidly toward smaller field strengths below its maximum. Possibly, this decrease is purely due to the limited Zeeman sensitivity of roughly $200-300 \mathrm{G}$ of the IR lines in the present observations. 

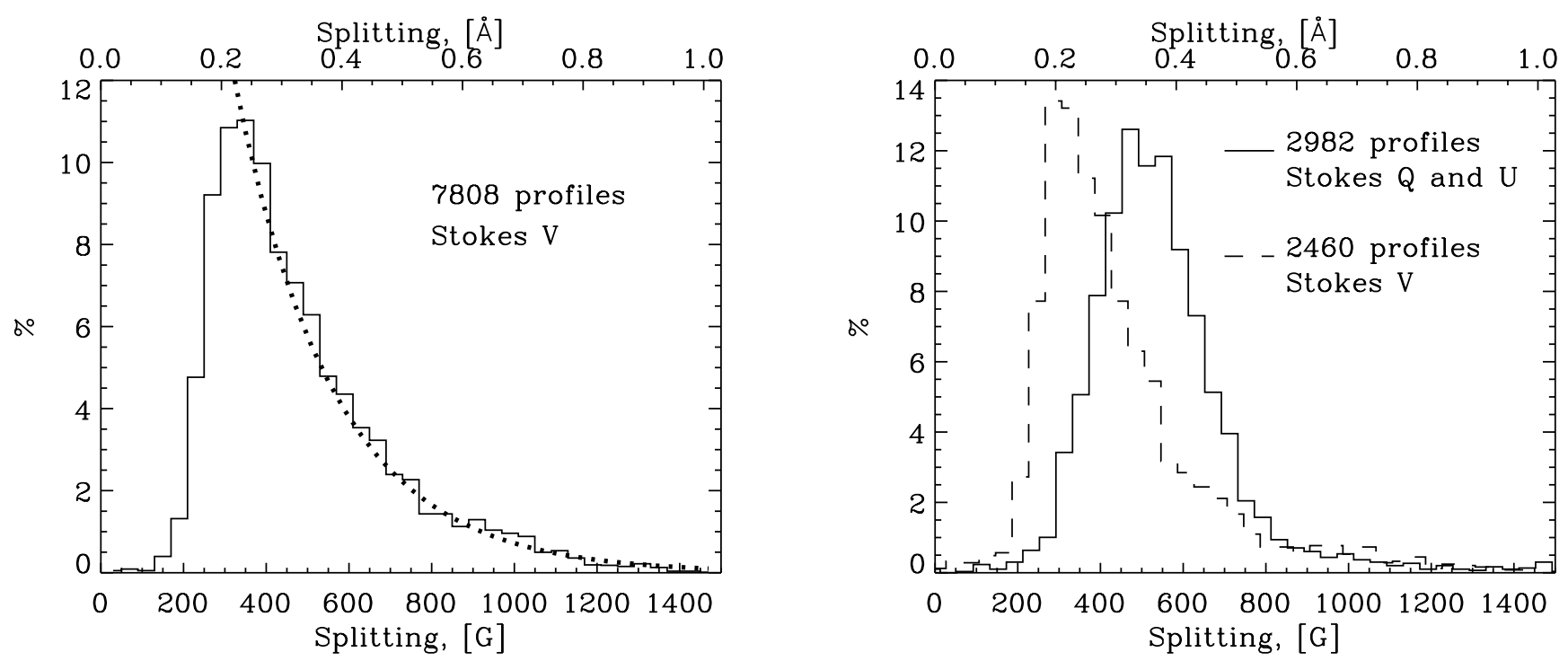

Fig. 6. Histograms of Zeeman splitting. Left panel: Stokes $V \sigma$-component splitting. Only profiles of the 2-lobe classes 1, 2, 4, 6 from Fig. 2 are used. Dotted line: fit by an exponential function $A \exp \left(-B / B_{0}\right)$ to the right part of the histogram beyond the maximum. The upper horizontal axes indicate the splitting in $\AA$, the lower axes in $\mathrm{G}$ (i.e. converted into field strength). Right panel: Stokes $Q$ and $U \sigma$-component splitting. Only classes 1, 2, 3 from Fig. 4 are used. Dashed line: Histogram of Stokes $V$ splitting for the pixels where a significant signal in Stokes $Q$ and/or $U$ is present.
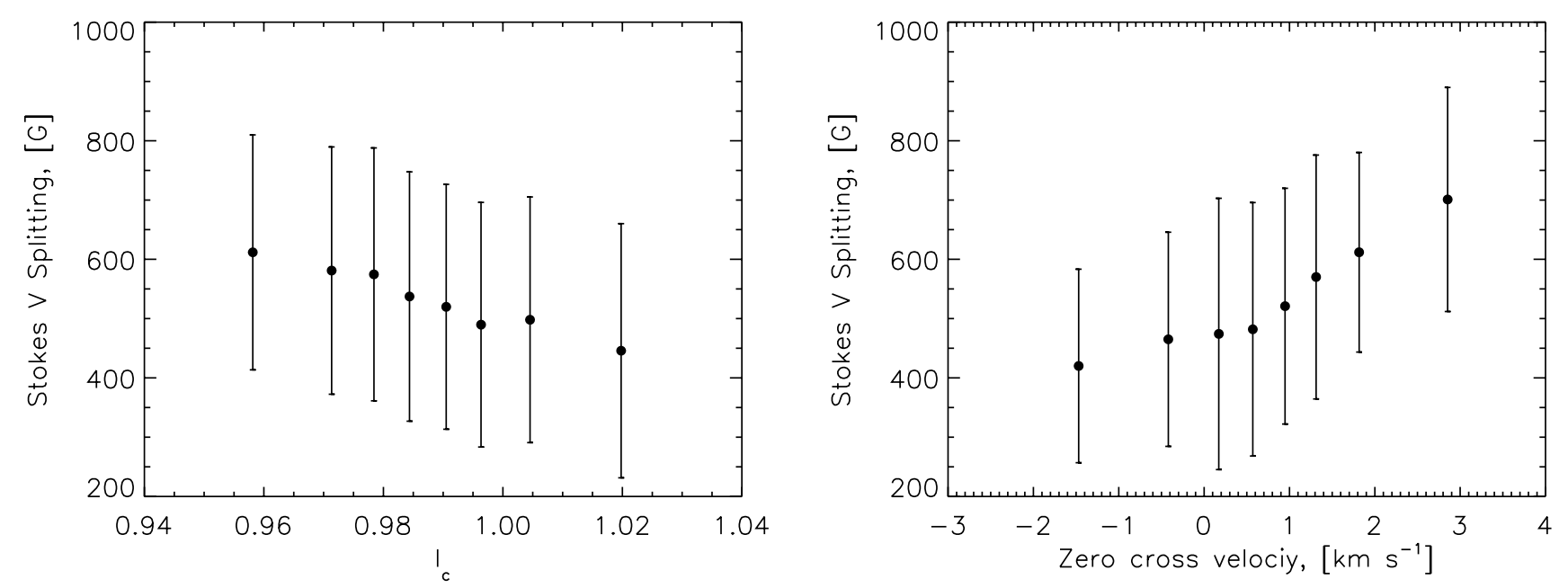

Fig. 7. The Stokes $V$ splitting as a function of continuum intensity (left panel) and Stokes $V$ zero crossing velocities (right panel). 7808 profiles from Stokes $V$ classes 1, 2, 4, 6 are used. The values of the splitting are binned into 9 intervals containing an equal number of points. Error bars show the standard deviation within each interval. Zero crossing velocities are measured with respect to the wavelength of the minimum Stokes $I$.

The number of pixels with a given magnetic field strength increases as its value decreases. The right part of the histogram beyond the maximum is well represented by an exponential function, $A \exp \left(-B / B_{0}\right)$ (shown by the dotted line in the left panel of Fig. 6) with $B_{0}=250 \mathrm{G}$. A significant fraction of the field $(40 \%)$ is below $400 \mathrm{G}$.

The histogram of the Stokes $Q$ and $U \sigma$-components splitting does not reach such low values as for Stokes $V$ (right panel of Fig. 6). It has its maximum at about $500 \mathrm{G}$ and has fewer points at $350 \mathrm{G}$. The dashed line in the right panel of Fig. 6 represents the histogram of Stokes $V$ splitting taken at the points where the signal in Stokes $Q$ or $U$ is above the threshold. This histogram is very similar to the one over all Stokes $V$ profiles. Hence, the field strength at the pixels with strong Stokes $Q$ and $U$ signal is not higher than the average. The reason for the difference of the histograms can reside in the different splitting regimes for Stokes $V$ and Stokes $Q$ and $U$. Figure $6 \mathrm{~b}$ thus supports the interpretation that the peak at $350 \mathrm{G}$ in Fig. 6a is not solar, but rather corresponds simply to the minimum field strength detectable by the Gaussian fitting. It suggests that not too much significance should be attached to the cut-off field strength in Fig. 6a.

Consider now the dependence of the field strength on the granulation pattern. We found hints that the splitting gets larger in the dark intergranular lanes. The left panel of Fig. 7 demonstrates this result. Here, the continuum intensity (Stokes $I$ ) was measured at the points having an appreciable signal in Stokes $V$. Only the July dataset was used because the seeing 


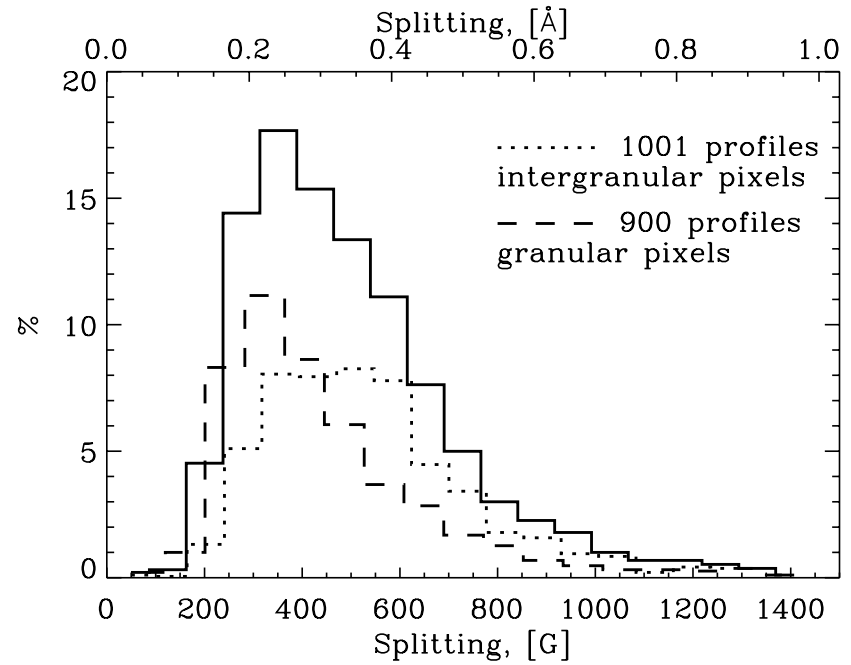

Fig. 8. Histograms of Zeeman splitting. Only profiles of the 2-lobe classes 1, 2, 4, 6 from Fig. 2 of the July data are used. The format of the figure is the same as Fig. 5. Solid line - histogram over all the points. Dotted line - only Stokes $V$ from the pixels with $I_{\mathrm{c}}<1$ (intergranular lanes). Dashed line - only Stokes $V$ from the pixels with $I_{\mathrm{c}}>1$ (granules).

was better. The data from September show a similar but weaker trend. All the data points in Fig. 7 were binned with an equal number of points (about 240) per bin. In spite of the large standard deviation, the binned data show a clear trend: the field increases from about $450 \mathrm{G}$ to $600 \mathrm{G}$ as the continuum intensity of the feature decreases from 1.02 to 0.96 . Another indication of the enhancement of the field in intergranular lanes is that there are more data points at the darker pixels $\left(I_{\mathrm{c}}<1\right)$. Since we only consider pixels with the amplitude of the signal in Stokes $V$ above the threshold, it means there is a less intense magnetic field at the bright granular pixels. On average, the continuum level of the pixels with the largest magnetic field strength is lower than the value averaged over the whole image.

The Stokes $V$ splitting also increases with increasing zerocrossing velocity, i.e. the field is stronger where the downflow is stronger. The right panel of Fig. 7 shows this dependence. The field is more intense when the flow of material is downward and is weaker when the flow is upward.

Further evidence for the presence of more intense fields in intergranular lanes is given in Fig. 8. Here we show histograms of the magnetic field strength in bright granular pixels $\left(I_{\mathrm{c}}>1\right)$ and dark intergranular pixels $\left(I_{\mathrm{c}}<1\right)$, together with the histogram over all the pixels in the July data. The histogram of the magnetic field strength in granules has a similar shape as the histogram over all the points. It peaks at about $300 \mathrm{G}$ and drops rapidly toward lower field strength values. Instead, the histogram of the field strength in intergranular pixels peaks at about $500 \mathrm{G}$ and has no such rapid cutoff. The position of the maximum in this case is not affected by the limited Zeeman sensitivity of the employed IR lines. Thus, on average, the magnetic field is stronger in intergranular lanes with the maximum of the distribution at $500 \mathrm{G}$ and both higher and lower values also being present.

\subsection{Magnetic field inclination}

In contrast to the $\mathrm{kG}$ fields of plages and network regions, which are oriented mostly vertically (e.g. Bernasconi et al. 1995; Martínez Pillet et al. 1997), very little is known about the inclination of the magnetic fields of the inter-network plasma. Nonetheless, patches of horizontal weak fields were found by Lites et al. (1996) and indications for a distribution of field inclinations were obtained from centre-to-limb variations of Stokes $V$ amplitudes by Martin (1988) and Meunier et al. (1998). Recently Lites (2002) argued from the analysis of data obtained in the Fe I lines at $\lambda 6302$ that, due to the insignificant amount of the detected linear polarization, it is hardly possible to deduce the inclination of the observed fields. The $15648 \AA$ line is a good diagnostic of the magnetic field inclination since the ratio of the $Q$ and $U$ amplitudes to $V$ is relatively large compared to less Zeeman sensitive lines (Solanki et al. 1992).

We have applied the following approximate expression to estimate the inclination of the magnetic vector to the line of sight, $\gamma$ (which corresponds closely to the inclination to the vertical since the observed regions were located very close to the centre of the solar disc):

$\frac{A_{V}}{\sqrt{A_{Q}^{2}+A_{U}^{2}}}=\frac{\cos \gamma}{\sin ^{2} \gamma}$,

where $A_{Q}, A_{U}$ and $A_{V}$ are the amplitudes of the Gaussians obtained from the fit and not the directly measured amplitudes of the Stokes profiles themselves. $A_{Q}$ and $A_{U}$ correspond to the amplitudes of the Gaussian describing the $\pi$ component of Stokes $Q$ and $U$ and $A_{V}$ corresponds to the amplitude of one of the $\sigma$ component of Stokes $V$. This expression is only valid for a weak line (the two observed spectral lines are near this limit), or for line which is completely Zeeman split. For a weak line, the amplitude of the Stokes parameters $Q, U, V$ are proportional to the absorption coefficients $\eta_{Q, U, V}=\eta_{0} H_{Q, U, V}$. Adopting the notations from Stenflo (1994),

$H_{Q}=H_{\Delta} \sin ^{2} \gamma \cos 2 \chi$

$H_{U}=H_{\Delta} \sin ^{2} \gamma \sin 2 \chi$

$H_{V}=1 / 2\left(H_{+}-H_{-}\right) \cos \gamma$,

where

$H_{\Delta}=1 / 2\left[H_{0}-1 / 2\left(H_{+}+H_{-}\right)\right]$

$H_{q}=H\left(a, v-q v_{H}\right), q=0, \pm 1$,

$H_{q}$ are Voigt profiles. The procedure of the Gaussian fit gives us directly the amplitudes of a $\sigma$ component $H_{+}$for Stokes $V$ and $\pi$ component $H_{0}$ for Stokes $Q / U$ (multiplied by the corresponding functions of $\gamma$ and $\chi$ ):

$\left.A_{Q} \sim H_{0}\right|_{v=0} \sin ^{2} \gamma \cos 2 \chi$

$\left.A_{U} \sim H_{0}\right|_{v=0} \sin ^{2} \gamma \sin 2 \chi$

$\left.A_{V} \sim H_{+}\right|_{v=v_{H}} \cos \gamma$.

Thus, in the case of a weak line the ratio of the amplitudes of the Gaussians $A_{V} / \sqrt{A_{Q}^{2}+A_{U}^{2}}$ is independent of the field strength.

To check if the last statement is valid for the lines employed here we performed the following test calculations. We have synthesized the Stokes $Q, U$ and $V$ profiles of the 


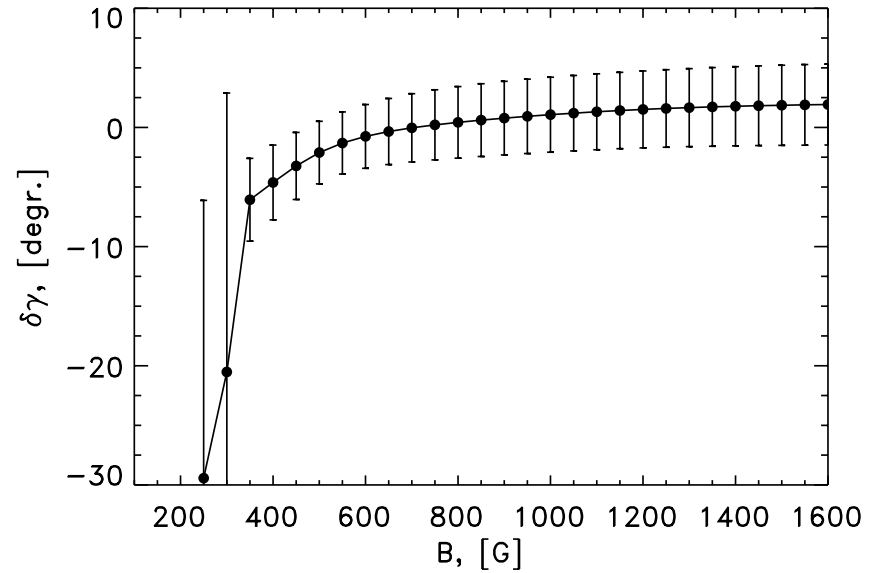

Fig. 9. Error of the derived inclination $\gamma$. Filled circles indicate values of $\delta \gamma=\gamma-\gamma_{0}$, averaged over the input values $\gamma_{0}$ ranging from $10^{\circ}$ to $80^{\circ}$. Values of the inclination $\gamma$ are inferred from the synthesized profiles using the relation (5) involving the amplitudes of the fitted Gaussians. Error bars show the standard deviation of $\delta \gamma$.

lines Fe I 15648 and $15653 \AA$ for a set of field strengths $B=100-1600 \mathrm{G}$ and a set of inclinations $\gamma_{0}=0-90$ degrees. To this end, we use the SIR code (Ruiz Cobo \& del Toro Iniesta 1992). The stratification of the atmospheric parameters was taken to be that of the quiet Sun (represented by the Harvard Smithsonian Reference Atmosphere of Gingerich et al. 1971) with no bulk flows along the LOS. The value of the macroturbulent velocity was taken equal $2 \mathrm{~km} \mathrm{~s}^{-1}$ and the microturbulent velocity was set to $0.6 \mathrm{~km} \mathrm{~s}^{-1}$ throughout the atmosphere. These values were chosen such that the width of the synthesized profiles is similar to the observed ones. We then applied the same procedure of the Gaussian fit to the synthesized profiles and inferred the inclination $\gamma$ using expression (5) as a function of the field strength. The results of this test are shown in Fig. 9. The error $\delta \gamma$ in the inclination remains within the range $\pm 2^{\circ}$ for values of the field strength from 500 to $1600 \mathrm{G}$. Thus the inclination derived from expression (5) suffers from a minute $B$-dependent error for $B>500 \mathrm{G}$. For field strengths from 350 to $500 \mathrm{G}$, the absolute value of $\delta \gamma$ is lower than $6^{\circ}$. The rapid increase of $|\delta \gamma|$ for $B<350 \mathrm{G}$ is due to the limitations of the Gaussian fit procedure. As was mentioned in Sect. 3.4, the sensitivity of the fit is limited by about 200-300 G for Stokes $V$, and by $350-450 \mathrm{G}$ for Stokes $Q / U$. Taking into account the results from Fig. 9, we restrict the profiles used for the determination of $\gamma$ from expression (5), to those with splitting larger than $350 \mathrm{G}$ for Stokes $V$ and $500 \mathrm{G}$ for Stokes $Q$ and $U$.

Figure 10 shows an estimate of the inclination of the field deduced for approximately $40 \%$ of all pixels in our data having an appreciable signal in either Stokes $Q, U$ or $V$. The distribution of the inclination over the observed region is presented in Fig. 5b. At the spatial points where the signal in the original data was below $10^{-3}$ (our amplitude threshold), we set the amplitude $(Q, U$ or $V$ in 5$)$ equal to the noise $2 \times 10^{-4}$. It must be noted that the noise level does not allow inclinations below about 10 degrees and larger than 85 degrees to be measured. This explains the absence of such points in Figs. 5 and 10.

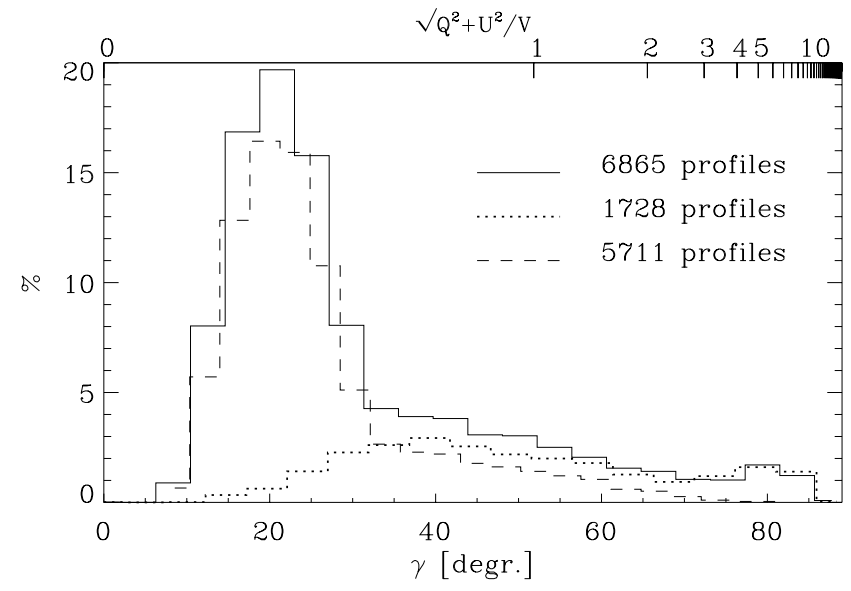

Fig. 10. Histogram of the ratio of Stokes $Q$ and $U$ amplitudes to Stokes $V$ amplitude. The values of the amplitudes of the Stokes parameters were taken from the Gaussian fit to the profiles. The horizontal upper axis denotes the amplitude ratio, and the lower axis the values of the inclination angle derived from this ratio. Solid line: histogram over all points where signal in at least one of the Stokes $Q, U$ or $V$ profiles is above the noise level. Dashed line: histogram over points where signal in Stokes $V$ exists. Dotted line: histogram over points where signal in Stokes $Q$ and/or Stokes $U$ exists.

The map presented in Fig. 5b shows that there is a significant amount of pixels with field lines that are rather inclined (marked by orange and yellow colours). The patches of horizontal fields occur in between the regions with a relatively vertical fields and also in isolation. There is a tendency for the patches with a relatively strong field to have it close to the vertical in the center and more inclined at the borders.

The histogram of inclinations in Fig. 10 over the points with signal in either $Q, U$ or $V$ has two maxima (solid line). It shows that most of the magnetic fields are oriented nearly vertically, especially in the intergranular regions. The histogram maximum is biased towards larger inclination values due to the noise. We expect that in reality this maximum is smeared out both towards smaller as well as larger inclinations. There is a secondary maximum for the more horizontal fields because of the significant number of pixels having a signal in $Q$ and $U$, but not in $V$ (patches of horizontal fields in Fig. 5). The fraction of magnetic elements with inclinations above $70^{\circ}$ is $5 \%$. Note that the $\gamma$ values of these points are definitely lower limits, not just because of the approximation underlying Eq. (5), but also because the $V$ amplitudes may also be significantly below the noise level. If we exclude such pixels (dashed-line histogram), the maximum at 80 degrees disappears. In the opposite case, if we keep only pixels with the signal in $Q$ and/or $U$ irrespective of whether a signal in $V$ is present or not, the histogram has no maximum at 20 degrees (dotted line). Instead, there is a secondary maximum in the dotted-line histogram at about 40 degrees.

Our estimations of the inclination may be affected by unresolved polarities that are possibly present in a pixel. This would lead to a decrease of Stokes $V$ amplitudes relative to $Q$ and $U$ amplitudes and thus would introduce a bias into the derived inclinations (see the discussion by Lites 2002). Given all the 


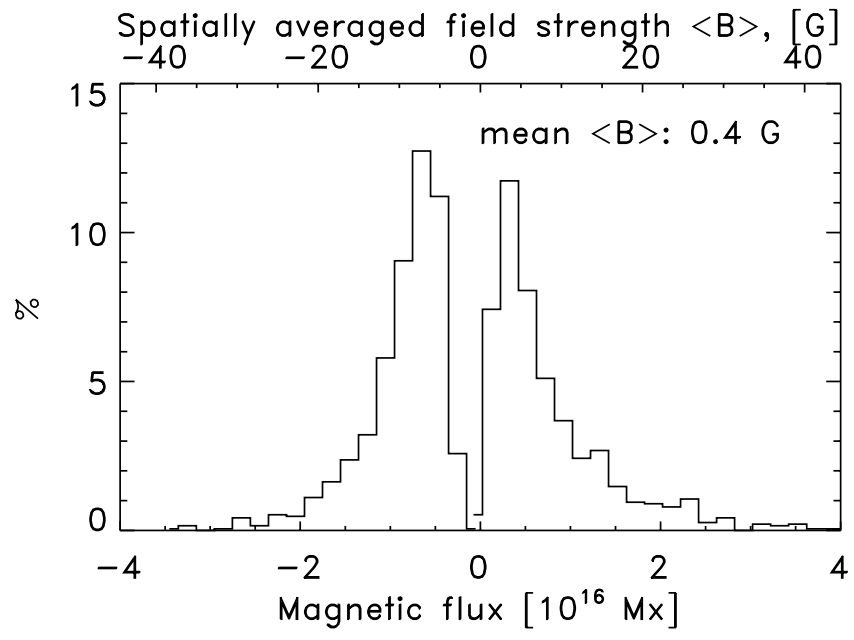

Fig. 11. Histogram of magnetic flux per pixel. Upper horizontal axis indicates values of the spatially averaged magnetic field strength per pixel. Lower horizontal axis indicates flux.

uncertainties, our estimates of the inclination should be considered as preliminary.

\subsection{Magnetic filling factor and flux}

Since the intrinsic magnetic field strengths are generally small, the magnetic pressure should produce only a small evacuation within the magnetic features and should also not decisively influence the dynamics of the plasma. Thus, we can assume that the thermodynamic properties of the atmosphere inside and outside of the magnetized elements are the same, and can estimate the filling factor from the following relation:

$f=\frac{2 V_{0}}{\left(I_{\mathrm{c}}-I_{0}\right) \cos \gamma}$,

where $V_{0}$ is the amplitude of the $\sigma$-component taken from the Gaussian fit to the $V$ profile, $\gamma$ is the inclination angle, obtained from relation (5), $I_{\mathrm{c}}$ is the continuum intensity and $I_{0}$ is the minimum observed residual intensity. This relation is valid for a weak line. The values of the filling factor obtained range up to $8 \%$, but most of them are lower than $2 \%$. This is not surprising since already the small Stokes $Q, U$ and $V$ amplitudes (see Sect. 3) indicate a very low filling factor of the observed fields. The obtained values can be an underestimate due to the presence of unresolved polarities in the pixel (see Sect. 3.5). However, it is difficult to estimate the importance of this effect with the present data.

Assuming that the $f$-values are essentially correct, we can obtain the flux from the relation:

$\phi=f R^{2} B \cos \gamma$,

where $R^{2}$ is the area of our resolution element $\left(0.38^{\prime \prime} \times 0.38^{\prime \prime}\right)$ and $B$ is the magnetic field strength. The histogram of the magnetic flux (including its sign) is given in Fig. 11. The upper horizontal axis of the figure indicates the values of the average magnetic field strength per pixel, defined as the value of the flux divided by the area of a pixel $(\langle B\rangle=f B \cos \gamma)$. The flux values are the lowest observed so far with the help of the Zeeman effect. The lower limit in the flux detection from our observations is about $2 \times 10^{15} \mathrm{Mx}$ per pixel implying a limit for the absolute value of $\langle B\rangle$ of $2 \mathrm{G}$. About $40 \%$ of the magnetic elements have absolute values of the flux lower than $5 \times 10^{15} \mathrm{Mx}$ per pixel, which is the flux detection limit of the data used by Lin $\&$ Rimmele (1999). The average value of the unsigned $\langle B\rangle$ over all pixels with significant $V$ signal in the observed area is $8 \mathrm{G}$ and the maximum of its distribution is at about $4 \mathrm{G}$. The similarly averaged unsigned net flux in the region is $7.5 \times 10^{15} \mathrm{Mx}$.

Assuming that in the pixels with amplitudes below the threshold the flux is at most $2 \times 10^{15} \mathrm{Mx}$ (our flux detection limit) and taking into account that we detect signals in $50 \%$ of all the pixels, we obtain that we have detected at least $75 \%$ of the magnetic flux that is in principle detectable with our spatial resolution of about $1^{\prime \prime}$. It is interesting to note that the positive and negative fluxes in the observed region are almost balanced. The average value of $\langle B\rangle$ including its sign is about $0.4 \mathrm{G}$ meaning that the net flux through the region amounts to $5 \%$ of the total flux.

Figures 5c and 5d show maps of the filling factor and the flux. The patches of enhanced flux generally coincide with the patches of large filling factor, but do not always coincide with the patches of strong Stokes $V$ splitting. The patches normally have large filling factor (flux) in the center and smaller values at the border.

The scatter plot of the splitting vs. flux is given in the left panel of Fig. 12. Figure 12 is similar to the results by Lin (1995) and Solanki et al. (1996), although the dependence of field strength on flux is marked somewhat by the rapid rise of scatter of magnetic field strength with flux. To better demonstrate the increase of the intrinsic field strength with increasing flux in the pixel we also plot binned values (right panel of Fig. 12). Assuming the average value of the filling factor to be $f=2 \%$, we obtain an average size of the magnetic elements of about $40 \mathrm{~km}$. This value is half that obtained by Lin \& Rimmele (1999). However, the uncertainties in the determined filling factor directly affect the radius, so that this result is considerably less reliable than those relating to the flux.

\subsection{Line-of-sight velocities}

We determined velocities (corresponding to macroscopic mass motions) from 3 observed parameters: the wavelength position of the zero crossing of Stokes $V, v_{\mathrm{zc}}$, the position of the maximum of the $\pi$-component of Stokes $Q$ and $U, v_{q u}$, and the position of the Stokes $I$ minimum $v_{I}$. We computed the last of these parameters as the first moment of the Stokes $I$ profile. Since the magnetic filling factor in our data is very small (on average $2 \%$ ), the velocity obtained from Stokes $I$ basically refers to the atmospheric regions which do not contribute significantly to polarization signals in the $1.56 \mu \mathrm{m}$ lines, while $v_{\mathrm{zc}}$ and $v_{q u}$ refer to the magnetized gas. All the shifts were measured with respect to the average wavelength position of the Stokes I profiles (see Sect. 2). The map of the $v_{\mathrm{zc}}$ velocities is displayed in Fig. 5e. Areas with redshifted $V$ profiles are marked by red and yellow colors. Comparing with the map of the magnetic field strength (Fig. 5a) it can be seen that at the centers of patches with enhanced field the velocity is usually a downflow, while 

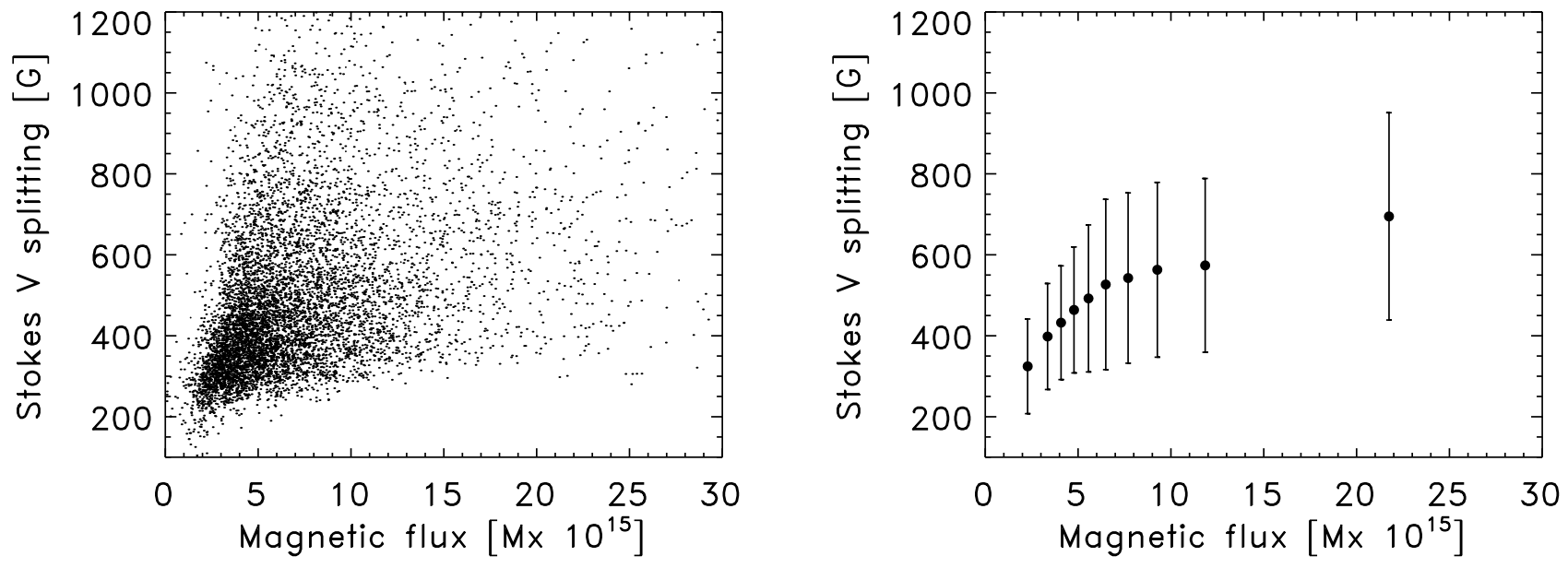

Fig. 12. Left panel: scatter plot of the splitting vs. magnetic flux. Right panel: the same, but for binned values.

at the edges it can become an upflow. Regions with upflows or small velocities coincide with the regions of weak fields, due to the correlation between the magnetic field strength and $v_{\mathrm{zc}}$, as demonstrated in Fig. 7.

Figure 13 shows the histograms of $v_{\mathrm{zc}}, v_{q u}$ and $v_{I}$ (from top to bottom). The $v_{\mathrm{zc}}$ histogram is asymmetric and shifted such that the mean value of the velocity is $550 \mathrm{~ms}^{-1}$ (a positive value implying a redshift). Since the shifts were measured with respect to the minimum intensity position at the quiet pixels which contains a residual of the convective blue shift, the actual Stokes $V$ redshift should be considerably lower. The width of the distribution is large, as the velocities vary from -3 to $5 \mathrm{~km} \mathrm{~s}^{-1}$. We believe this relatively large scatter of the velocities is not an artifact due to noise. If it were an artifact there should be an anti-correlation between the shift and the amplitude of the signal. This anti-correlation is absent. We find no difference in the $v_{\mathrm{zc}}$ distribution if we only consider those pixels with significant $Q$ and $U$ (dashed line). From observations in a quiet solar region Grossmann-Doerth et al. (1996) found an average $V$ zero-crossing velocity of $970 \mathrm{~m} \mathrm{~s}^{-1}$ (relative to the position of the centre of the spatially averaged Stokes $I$ profile) with a broad distribution of positive and negative shifts increasing toward the weakest $V$ signals. An average redshift of $730 \mathrm{~ms}^{-1}$ (after correction for convective blueshift) was also found by Sigwarth et al. (1999) for a quiet region. These results refer mainly to network elements and can be interpreted as the presence of net flows inside flux tubes, in rough agreement with numerical simulations (Grossmann-Doerth et al. 1998), suggesting a strong dynamical behaviour of magnetic elements. Our results, which show a much smaller average redshift, indicate that the IN features have a different internal velocity structure than the network elements studied by Grossmann-Doerth et al. (1996) and Sigwarth et al. (1999). The fact that $V$ profiles with larger splitting exhibit a stronger redshift (Figs. 5 and 7) suggests that the difference between the average shifts of network and inter-network is related to the difference in field strength between the two types of features.

The $v_{q u}$ exhibit a smaller spread of $\pm 1.5 \mathrm{~km}$ (middle panel of Fig. 13). Contrary to the values obtained for the $V$ zero-crossing velocities, $v_{q u}$ shows no net shift: the maximum of this histogram is around zero. The smaller spread of $v_{q u}$ values compared to $v_{\mathrm{zc}}$ suggests that the major component of the velocity field is directed along the magnetic field lines. Nonetheless, the non-negligible $v_{q u}$ values imply that the relatively horizontal fields do appear to move vertically in the atmosphere in a random fashion. Because of the residual blueshift in the $I$ profile, the average $v_{q u}$ corresponds to an upflow.

Due to the small filling factors (calculated above), the intensity profile comes mainly from the non-magnetic surroundings of the magnetic features. The histogram of the Stokes $I$ velocities taken at the pixels with no magnetic signal above the threshold is symmetric and is centered around zero, which is not surprising, given the fact that the "rest" wavelength we compare with was determined this way. The maximum of the histogram of the Stokes $I$ velocities at the pixels with magnetic field above $500 \mathrm{G}$, however, shows a redshift of $110 \mathrm{~m} \mathrm{~s}^{-1}$. This redshift is consistent with the correlation between $v_{\mathrm{zc}}$ and Stokes $I$ velocities (Fig 14).

Figure 14 shows the correlation between the velocities and their dependence on the continuum intensity. The correlation between $v_{\mathrm{zc}}$ and $v_{I}$ is positive (Fig. 14 top left), so that the downflows within flux tubes are accompanied by downflows in the surrounding atmosphere. When the $v_{I}$ corresponds to an upflow, the corresponding value of $v_{\mathrm{zc}}$ is close to zero. Only binned values have been plotted in order to show the trend more clearly. The error bars indicate the standard deviation of the individual points within the bin.

There is a weak correlation $(25 \%)$ between velocities from linear and circular polarized spectra (Fig. 14 top right). These velocities have the same sign for downflows, whereas if an upflow is observed in Stokes $Q$ and/or $U$, the average $v_{\text {zc }}$ velocity is close to zero. However, the weakness of the correlation (due to the large standard deviations) indicates that in many cases the velocities from $V$ and $Q / U$ spectra are not the same. It means that many pixels have unresolved magnetic structure inside, with a magnetic field having at least different inclinations within them. These different components are associated with different velocities. 

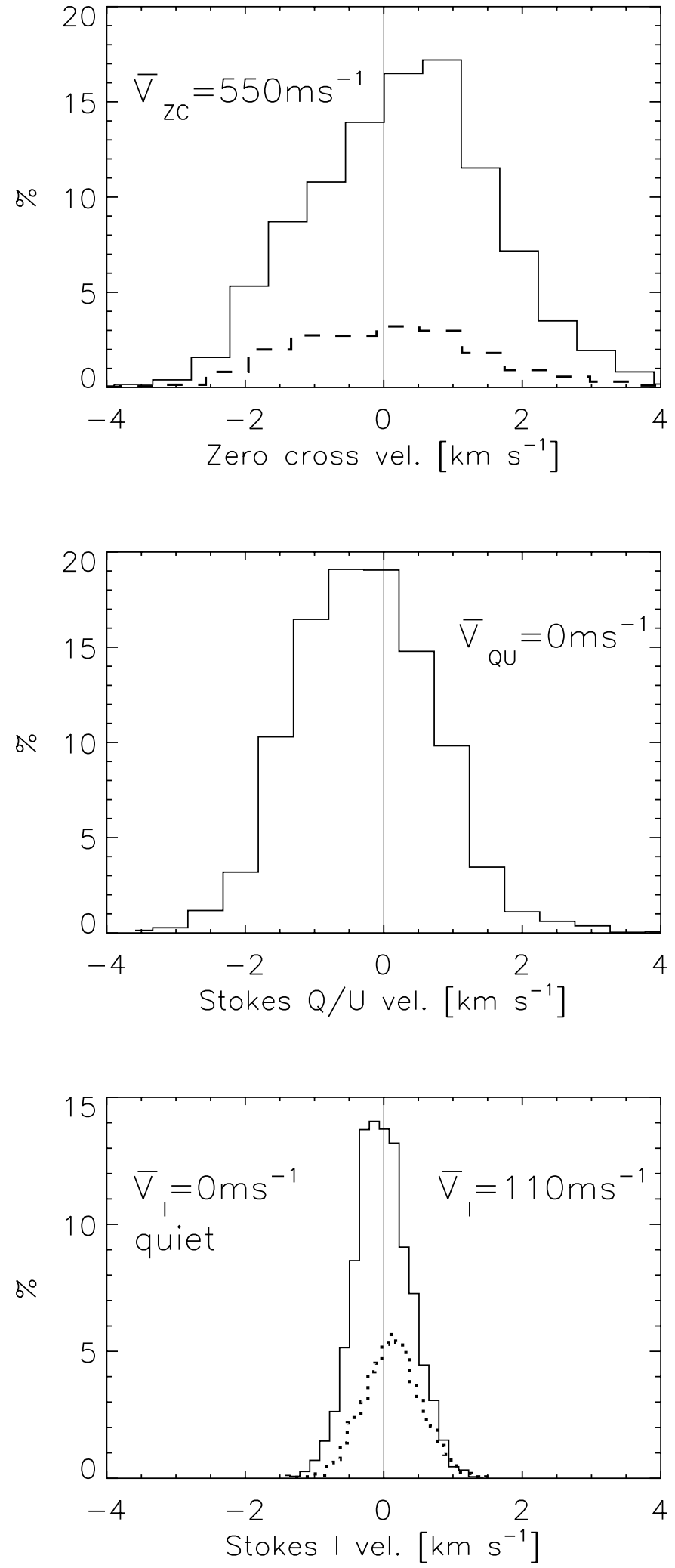

Fig. 13. Histograms of the line-of-sight velocities in the magnetized and in the (nearly) field-free atmospheres. Top panel: Stokes $V$ zerocrossing velocity. Solid line: all $V$ profiles. Dashed line: zero-crossing velocities at the pixels where signal in Stokes $V$ is accompanied by a signal in Stokes $Q$ and/or $U$. Middle panel: histogram of the Stokes $Q$ and $U \pi$-component velocities. Bottom panel: LOS velocities from Stokes $I$. Solid line: only pixels with the magnetic signal below the threshold were used. Dotted line: only pixels with the splitting in Stokes $V$ larger than $500 \mathrm{G}$ were used.
The correlation between $v_{I}$ and continuum intensity is typical for the granulation pattern (Fig. 14 bottom left). Only pixels without magnetic signal were included in this plot. The $v_{\mathrm{zc}}$ shows a similar dependence on $I_{\mathrm{c}}$, with almost all binned points being downflows (Fig. 14 bottom right). The $v_{\text {zc }}$ above granules are close to zero (implying a small blueshift, given the granular blueshift of the $I$ profile). It can be that the magnetic field lines are strongly inclined above the granules as suggested by MHD simulations (see Gadun 2000; Gadun et al. 2001).

\subsection{Asymmetries}

Figure 15 shows histograms of asymmetries of Stokes $V$ (top 2 panels) and Stokes $Q$ and $U$ (bottom panel) (see also the map of the asymmetries in Fig. 5f). They are defined in the usual way, $\delta a=\left(a_{b}-a_{r}\right) /\left(a_{b}+a_{r}\right)$, where $a_{b, r}$ are the absolute values of either the amplitude or the area of the $\sigma$-component of Stokes $V$ or the amplitude of the $\sigma$-component of Stokes $Q$ or $U$ (Solanki \& Stenflo 1984; Solanki et al. 1987). The amplitudes and areas in this case were determined directly from the profiles. The range of $V$-amplitude asymmetries is very wide, varying from $-70 \%$ to $+70 \%$. The same is true for the Stokes $Q$ and $U$ asymmetries, which display an even broader histogram. However, in this case the influence of the noise due to the smaller values of the amplitudes probably contributes to the width of the histogram. There is a strongly increased scatter of the asymmetry values toward smaller values of the amplitudes, which is consistent with the influence of noise. Note that if we include the "abnormal" profiles from classes 3, 5, 7 and 8 then large asymmetry values (over $100 \%$ ) are even more common.

The distribution of the area asymmetry of Stokes $V$ is narrower. The average values of the asymmetries (given in the plot) are positive in all cases and coincide rather well with the values published by Sigwarth et al. (1999) and Grossmann-Doerth et al. (1996) for Fe I 6301 and $6302 \AA$ in a quiet network region. In network and plages, the asymmetry of Fe I $15648 \AA$ is on the whole quite small (Stenflo et al. 1987). This is compatible with the fact that very large velocity gradients are needed to produce an asymmetry in a strongly split line (Grossmann-Doerth et al. 1991). Since we are dealing with less strongly split profiles the observed range of asymmetries is probably reproducible by similar mass motions with correlated magnetic vector and velocity gradients as lines in the visible (see Solanki 1993, and references therein).

There are indications for a dependence of the asymmetries on zero crossing velocity (Fig. 16, compare also the $v_{\mathrm{zc}}$ and asymmetry maps given in Fig. 5e and 5f). The effect is different for the different classes of Stokes $V$ profiles. The amplitude asymmetry of narrow profiles with a small Zeeman splitting from classes 2, 3 and 5 show a strong dependence on the velocity inside the magnetic element. The positive asymmetry increases with increasing speed of the downflows. The asymmetry of classes with larger splitting, however, shows a much weaker dependence (lower panels of Fig. 16). In all cases, no correlation between area asymmetry and $v_{\mathrm{zc}}$ is found. The correlation between the area and the amplitude asymmetries is 

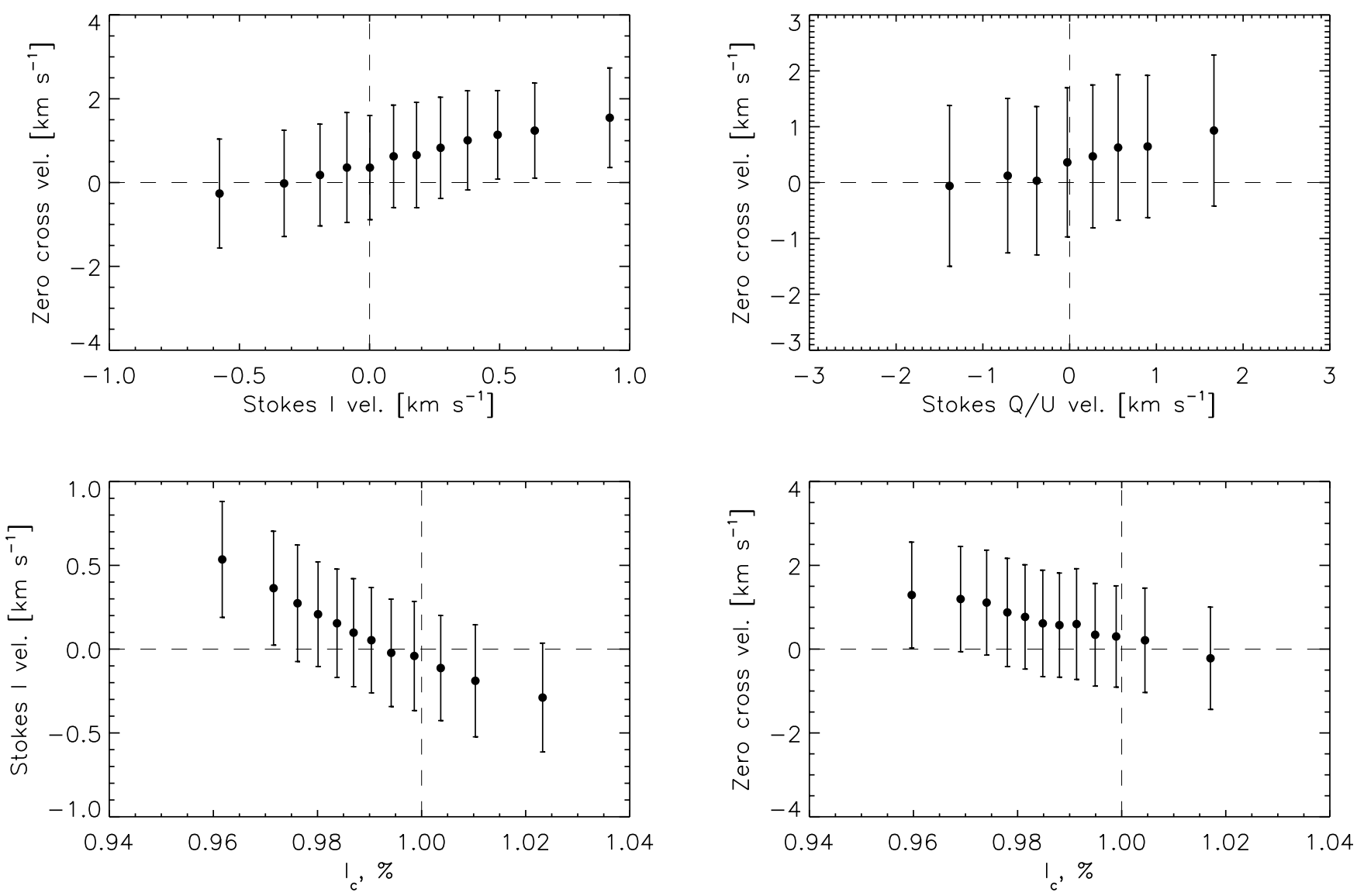

Fig. 14. Velocity correlations. Top left: $v_{\mathrm{zc}}$ vs. $v_{I}$. Solid points are binned values. Error bars indicate standard deviation. Top right: $v_{\mathrm{zc}}$ vs. $v_{q u}$. Bottom left: $v_{I}$ velocities vs. continuum intensity at the non-magnetic pixels. Bottom right: $v_{\mathrm{zc}}$ vs. continuum intensity $I_{\mathrm{c}}$. Only the July dataset was used for this plot due to the better seeing conditions.

positive, but the scatter of the data points is quite large. Here the correlation is better for the classes with stronger splitting. The large scatter can be a consequence of finite spatial resolution producing averaging over many magnetic elements in both horizontal and vertical directions (Sheminova 2002). The spatial averaging affects more the amplitude asymmetry than the area asymmetry. As a consequence, the amplitude asymmetry becomes larger than the area asymmetry and the correlation between these two quantities decreases (Sheminova 2002).

\section{Discussion and conclusions}

High-resolution, low-noise spectropolarimetric observations in the infrared at $1.56 \mu \mathrm{m}$ obtained with the Tenerife Infrared Polarimeter (TIP) have allowed us to analyze statistically a weak low-flux component of the magnetic field in two quiet Sun regions (inter-network fields far away from the network). We can summarize our results as follows:

- Most of the intrinsic field strength distribution in the IN, that we are able to detect using the IR lines at $1.56 \mu \mathrm{m}$, is well below $\mathrm{kG}$. The histogram of the field strength has an exponential shape and peaks at $350 \mathrm{G}$. Very likely, this peak is artificial since it is very close to the lowest measurable field strength (near $300 \mathrm{G}$ ) and may well just be the result of this cutoff. Only $4 \%$ of the profiles in our data show a direct splitting larger than $1 \mathrm{kG}$. However, some of the Stokes- $V$ profile classes we have found (e.g. class 6) might well be the result of the coexistence of a weak-field and a strong-field magnetic component with the same polarity (see below).

- A clear correlation is found between the field strength and the granulation continuum intensity and velocity, in the sense that strong fields tend to be located in intergranular lanes. Hence, the field amplification occurs in intergranules with strong downflows.

- The average filling factor occupied by these weak fields in our data is $2 \%$. The magnetic fluxes are extremely low. In $90 \%$ of the pixels the flux is below $1.5 \times 10^{16} \mathrm{Mx}$. With our flux detection limit of about $2 \times 10^{15} \mathrm{Mx}$ per pixel, the lowest observed so far with the help of the Zeeman effect, we find magnetic fields in more than $50 \%$ of all the pixels. We find some evidence that we detect most of the net flux present on the Sun at the 1 "spatial resolution of the present observations.

- Although most of the detected fields have nearly vertical orientations (around 20 degrees from the solar radius vector), a significant fraction of the magnetic field lines are considerably inclined. In about $25 \%$ of the pixels with significant $Q, U$ or $V$ the field lines are inclined by more than $40^{\circ}$, and in about $5 \%$ by more than $70^{\circ}$. 

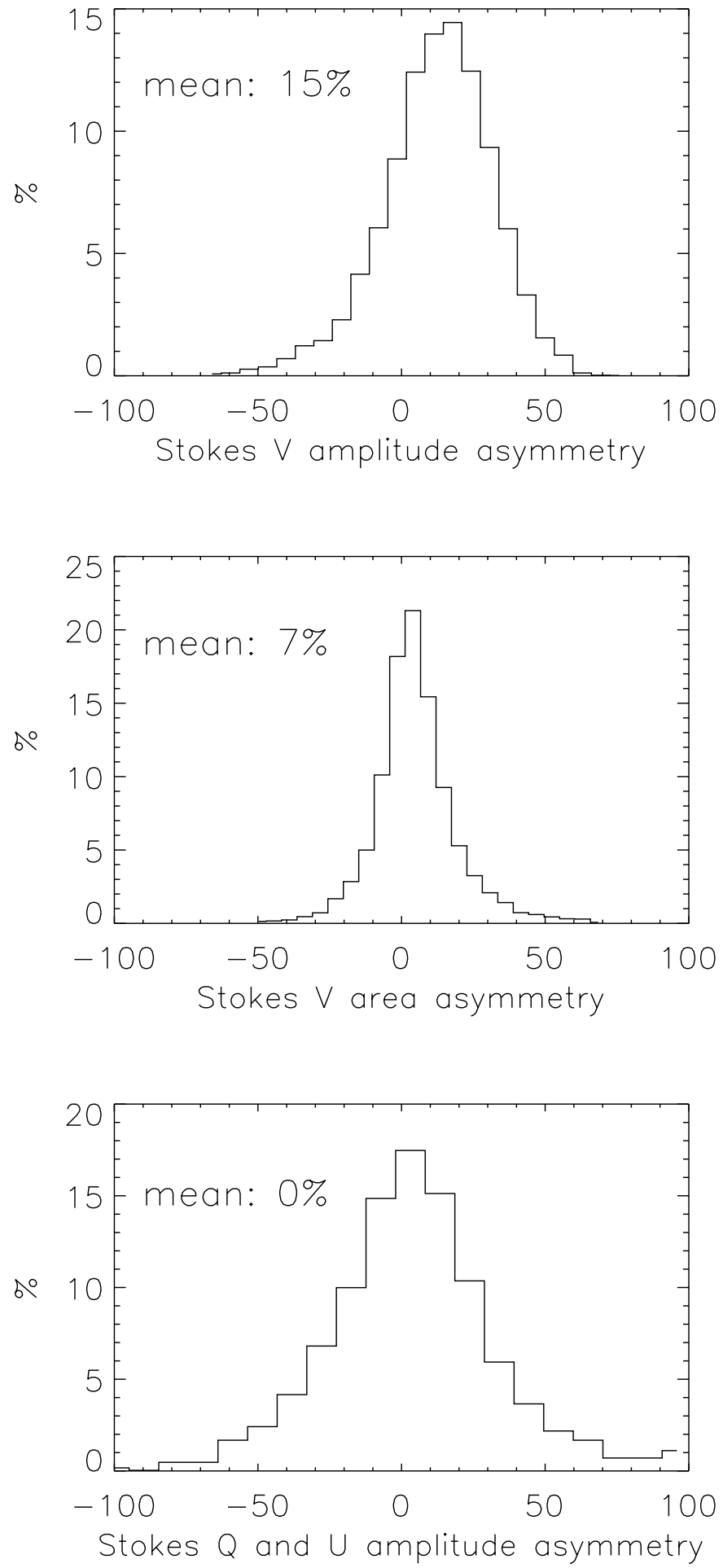

Fig. 15. Histograms of the amplitude and area asymmetries of the $\sigma$-components of Stokes $V$ (two upper panels) and amplitude asymmetry of the $\sigma$-components of the Stokes $Q$ and $U$ profiles (lower panel). The asymmetries are given in percent.

- Line-of-sight velocities in magnetic elements vary from $-3 \mathrm{~km} \mathrm{~s}^{-1}$ (upflow) up to $5 \mathrm{~km} \mathrm{~s}^{-1}$ (downflow). On average, the net flow is $550 \mathrm{~m} \mathrm{~s}^{-1}$ (relatively to the blueshifted $I$ profile), which is weaker than that observed for network areas.

- Most of the Stokes profiles are very asymmetric (in both amplitude and area), with both signs of the asymmetry being present. For the Stokes $V$ profiles positive asymmetries dominate. The Stokes $V$ asymmetries of weakly split profiles show a correlation with the Stokes $V$ zero-crossing. The asymmetry changes sign from negative to positive as the zero-crossing shift changes from an upflow to a downflow.

- Profiles of similar shape form patches on scales close to the granular spatial scales. As the seeing conditions improve the size of the patches decreases, suggesting that the characteristic scale of the magnetic fields in the inter-network is smaller than our spatial resolution.

- Stokes $V$ 3-lobe profiles not only occur between regions of changing polarity, but can form separate patches. The amount of mixed-polarity profiles decreases in the regions closer to the network (seen in the September data) and if the seeing conditions deteriorate.

We conclude that the observed magnetic field is highly dynamical with velocity and magnetic field gradients, with downflows and upflows and that there is a continuous distribution of small magnetic elements with different polarities and field strengths. The properties of the observed field are, in general, close to the "granular" magnetic fields seen by Lin \& Rimmele (1999), but the field strengths and fluxes obtained by us are lower. Our observations are at the limit of the sensitivity which can currently be reached with the Zeeman effect. They do not exclude the possibility that even weaker fields are present in between the observed magnetic flux concentrations, but such a "turbulent" mixed-polarity field cannot be investigated with the present technique. Fortunately, new diagnostic techniques based on the Hanle effect in molecular and atomic lines are facilitating a more reliable empirical investigation of the possibility of very weak "turbulent" fields in the solar photosphere (see Trujillo Bueno 2003). Such a weak-field component is speculated to exist mainly in the form of mixed polarities at scales well below $700 \mathrm{~km}$ on the Sun (e.g. Stenflo 1999). Since the magnetic features detected by us are concentrated in a small percentage of the area, a turbulent magnetic component could easily coexist with the IN fields. Indeed, we find evidence for multiple components even among the IN elements.

Most of the inter-network fields as detected directly from the observed splittings in the IR lines have strengths below kG. The improvement of the spatial resolution and the decreased noise in our observations relative to earlier works lead to lower fluxes, weaker field strengths and smaller sizes of the magnetic elements detected (compare with Lin 1995; Solanki et al. 1996; Lin \& Rimmele 1999). However, observations of quiet Sun regions using visible lines mainly show larger field strengths. Some authors (e.g. Sánchez Almeida \& Lites 2000) argue that the IR lines do not easily reveal the existence of $\mathrm{kG}$ fields because their Stokes $V$ profiles arising from pixels containing $\mathrm{kG}$ fields may have lower amplitudes (although large splittings) than for weaker fields. However, it is of interest to point out that our polarimetric sensitivity in the July data has been high 

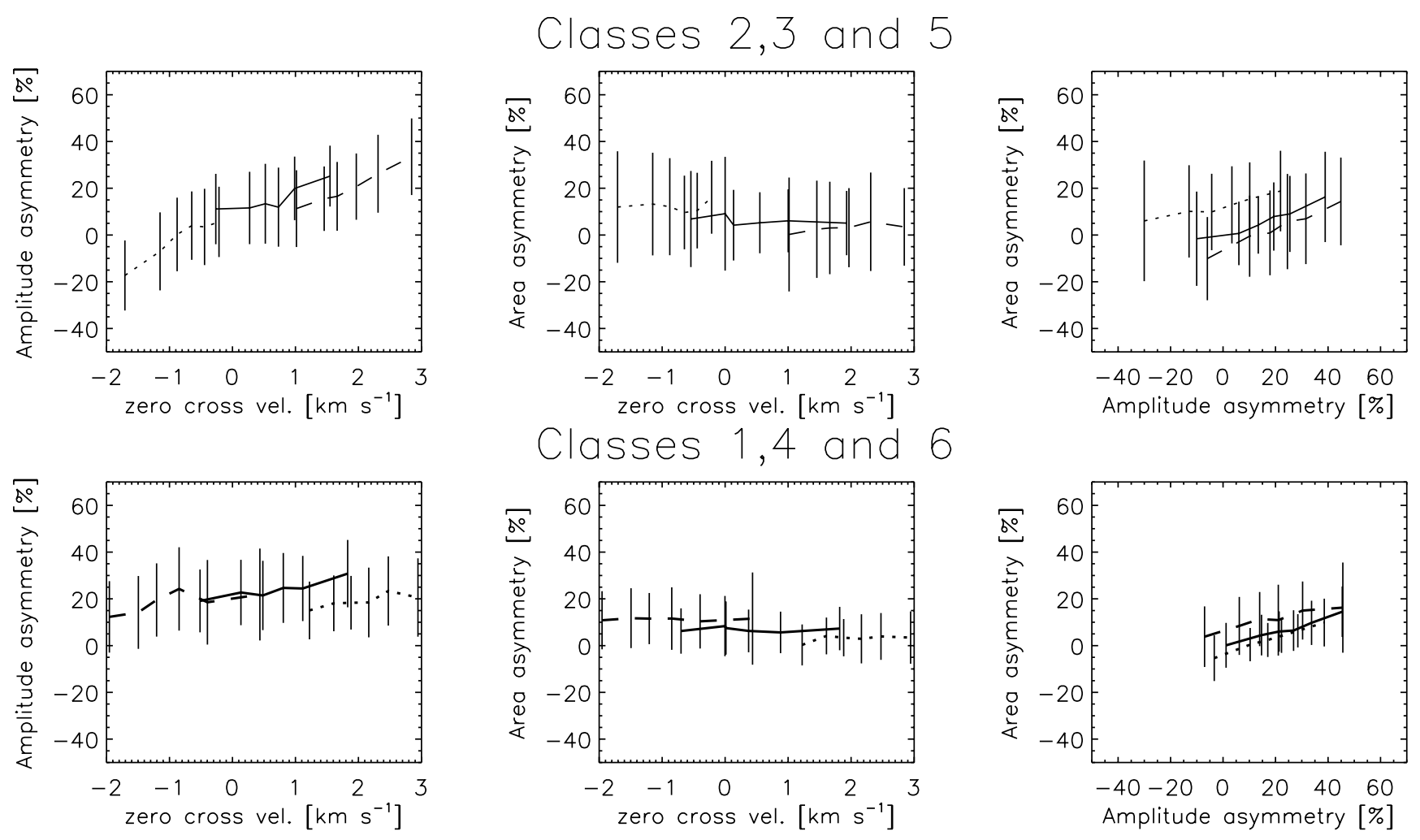

Fig. 16. Stokes $V$ amplitude and area asymmetries for different classes. The three top panels are for classes 2,3 and 5 from Fig. 2 . Solid line: class 2 , dotted line: class 3 , dashed line: class 5 . Top left: amplitude asymmetry vs. $v_{\mathrm{zc}}$, Top middle: area asymmetry vs. $v_{\mathrm{zc}}$, Top right: area asymmetry vs. amplitude asymmetry. Error bars indicate standard deviation of the data points. The three bottom panels are for classes 1,4 and 6 from Fig. 2. Thick solid line: class 1, dotted line: class 4, dashed line: class 6.

enough so as to detect the conspicuous Stokes $V$ profiles of class 6, with "bumps" in the red wings, which may well be the observational signature in the IR lines of the coexistence of both intrinsically weak and strong fields within a single resolution element. In fact, the synthetic Stokes $V$ profiles of IR lines obtained by Socas-Navarro \& Sánchez Almeida (2003) by combining weak and $\mathrm{kG}$ fields are very similar to our class 6 Stokes $V$ profiles $^{1}$. Thus, we conclude that most (but not all) of the inter-network pixels that give polarimetric signals above the noise level are due to weak fields with intrinsic strengths well below $\mathrm{kG}$.

Realistic magnetoconvection simulations are starting to become available (see the review by Schüssler 2001, and references therein). They show that the expulsion process effectively sweeps an initially homogeneous magnetic field (either unipolar or bipolar) into intergranular lanes where the flows converge leading to the field intensification (e.g. Grossmann-Doerth et al. 1998; Gadun 2000; Gadun et al. 2001). This mechanism produces field strengths roughly in equipartition with the convection, i.e. generally below $500 \mathrm{G}$, depending on the kinetic energy of convection and, thus, the height in the atmosphere.

Our observations are in agreement with the picture of the magnetic flux expulsion into downflow regions (see Fig. 7). There are less pixels with a significant Stokes $V$ signal within granules. It is either below the detection limit (histogram in

\footnotetext{
${ }^{1}$ Since the fits to these profiles are mainly dominated by the narrow, weakly split peaks, the fraction of strong fields admixtured to the weak may indeed be underestimated.
}

Fig. 8 shows that in granules the field is generally weaker), or it can be strongly inclined above granules, in agreement with MHD simulations. However, the estimated width of the concentrations formed, that is $40 \mathrm{~km}$, is smaller than the photon mean free path. Such weak field concentrations should have thermodynamical properties not very different from the surrounding atmosphere. Further intensification of the field according to the convective collapse instability (Parker 1978; Grossmann-Doerth et al. 1998) will not be effective for such small field dimensions and fluxes (Fig. 11). The amount of flux in IN magnetic elements as detected by us with the IR lines seem to be not sufficient to create stable $k G$ features. Previously, only a few examples were found of amplification of the field strength of a feature up to $\mathrm{kG}$ in inter-network (e.g. Lin \& Rimmele 1999; Bellot Rubio et al. 2001, however kG field strength was not reached in the last example).

There are different possibilities for the formation of IN fields. The first is that small loops of flux amplified by the main dynamo erupt all over the Sun. This would imply an enormous efficiency of this dynamo and is generally not considered realistic. Another possibility is the "recycling" mechanism pointed out by Ploner et al. (2001) to explain the high rate of flux emergence in the inter-network. The formation of a new intergranular lane often leads to a cancellation of magnetic elements of different polarities and flux disappearing and then appearing at a new place. This flux recycling may be registered as new flux. An alternative scenario is the local dynamo mechanism in the 
simulations by Cattaneo (1999) (see also Emonet \& Cattaneo 2001a,b). In these idealized simulations a random seed field is enhanced by dynamo action and turbulent motions concentrate the magnetic field into discrete structures that evolve with the granulation time scale. The effectiveness of the local dynamo action depends on the competition between the field amplification by magnetic field-line stretching and field destruction by enhanced dissipation (Cattaneo 1999). In contrast to the intensification of the field due to the convective collapse instability, where a certain amount of flux is needed for it to start working efficiently, the generation of the field by the dynamo action only happens for very weak fields, so that the turbulence dominates the dynamics. Emonet \& Cattaneo (2001a) indicate that this mechanism is able to generate also $\mathrm{kG}$ fields. In contrast, Nordlund \& Stein (2002) argue, on the basis of their recent realistic magneto-convection simulations, that, in the real stratified solar atmosphere, the surface layers do not play any energetically significant role and there is no specific local surface dynamo.

Our results (the weakness of the field, exponential shape of the histogram of the field strength distribution, dependence on granulation parameters, variations of inclination, spatial distribution of the field) point toward a chaotic character of the inter-network fields, intimately related to the granular motions. However, we cannot distinguish definitely which of the above mechanisms is mainly responsible for the field generation and intensification. Our empirical results, based on the Zeeman effect in IR lines, agree with the predictions of flux expulsion and "recycling" together with ineffective convective collapse taking place, but equally also with the flux expulsion mechanism associated with dynamo action at the granulation scales.

Acknowledgements. Helpful discussions with L. Bellot Rubio, J. Sánchez Almeida, V. Sheminova and H. Socas-Navarro are gratefully acknowledged. This work was partially supported by INTAS grant 00-00084 and by the Spanish Ministerio de Ciencia y Tecnología and by FEDER through project AYA2001-1649.

\section{References}

Ballesteros, E., Collados, M., Bonet, J. A., et al. 1996, A\&AS, 115, 353

Bellot Rubio, L. R., \& Collados, M. 2003, A\&A, in press

Bellot Rubio, L. R., Collados, M., Ruiz Cobo, B., \& Rodríguez Hidalgo, I. 2000, ApJ, 534, 989

Bellot Rubio, L. R., Rodríguez Hidalgo, I., Collados, M., Khomenko, E., \& Ruiz Cobo, B. 2001, ApJ, 560, 1010

Bernasconi, P. N., Keller, C. U., Povel, H. P., \& Stenflo, J. O. 1995, A\&A, 302, 533

Cattaneo, F. 1999, ApJ, 515, L39

Collados, M. 1999, in Solar magnetic fields and oscillations, ed. B. Schmieder, A. Hofmann, \& J. Staude, ASP Conf. Ser., 184, 3

Collados, M. 2001, in Advanced solar polarimetry: Theory, observation and instrumentetion, ed. M. Sigwarth, ASP Conf. Ser., 236, 255

Emonet, T., \& Cattaneo, F. 2001a, in Advanced solar polarimetry: Theory, observation and instrumentetion, ed. M. Sigwarth, ASP Conf. Ser., 236, 355
Emonet, T., \& Cattaneo, F. 2001b, ApJ, 560, L197

Frazier, E. N., \& Stenflo, J. O. 1972, Sol. Phys., 27, 330

Gadun, A. S. 2000, Kinematika Fiz. Nebesn. Tel, 16, 75

Gadun, A. S., Solanki, S. K., Sheminova, V. A., \& Ploner, S. R. O. 2001, Sol. Phys., 203, 1

Gingerich, O., Noyes, R. W., Kalkofen, W., \& Cuny, Y. 1971, Sol. Phys., 18, 347

Grossmann-Doerth, U., Keller, C. U., \& Schüssler, M. 1996, A\&A, 315,610

Grossmann-Doerth, U., Schüssler, M., \& Solanki, S. K. 1991, A\&A, 249, 239

Grossmann-Doerth, U., Schüssler, M., \& Steiner, O. 1998, A\&A, 337, 928

Harvey, L. W., \& Hall, D. 1975, BAAS, 7, 459

Keller, C. U., Deubner, F.-L., Egger, U., Fleck, B., \& Povel, H. P. 1994, A\&A, 286, 626

Lin, H. 1995, ApJ, 446, 421

Lin, H., \& Rimmele, T. 1999, ApJ, 514, 448

Lites, B. W. 2002, ApJ, 573, 431

Lites, B. W., Leka, K. D., Skumanich, A., Martínez Pillet, V., \& Shimizu, T. 1996, ApJ, 460, 1019

Livingston, W. C., \& Harvey, J. W. 1971, in The Magnetic and Velocity Fields of Solar Active Regions, ed. R. Howard (Dordrecht: Reidel), IAU Symp., 43, 51

Martin, S. F. 1988, Sol. Phys., 117, 243

Martínez Pillet, V., Lites, B. W., \& Skumanich, A. 1997, ApJ, 474, 810

Martínez Pillet, V., Collados, M., \& Sánchez Almeida, J., et al. 1999, in High resolution solar physics: theory, observations and techniques, ed. T. R. Rimmele, K. S. Balasubramaniam, \& R. R. Radick, ASP Conf. Ser., 183, 264

Meunier, N., Solanki, S. K., \& Livingston, W. C. 1998, A\&A, 331, 771

Nordlund, Å., \& Stein, R. F. 2002, in SOLMAG: Magnetic Coupling of the Solar Atmosphere Euroconference and IAU Colloq. 188, ed. H. Sawaya-Lacoste, ESA SP, 505, 83

Parker, E. N. 1978, ApJ, 221, 368

Ploner, S. R. O., Schüssler, M., \& Solanki, S. K. 2001, in Advanced solar polarimetry: Theory, observation and instrumentetion, ed. M. Sigwarth, ASP Conf. Ser., 236, 363

Rabin, D. 1992, ApJ, 391, 832

Rees, D. E., López Ariste, A., Thatcher, J., \& Semel, M. 2000, A\&A, 355,759

Ruiz Cobo, B., \& del Toro Iniesta, J. C. 1992, ApJ, 398, 375

Rüedi, I., Solanki, S. K., Livingston, W., \& Stenflo, J. O. 1992, A\&A, 263,323

Sánchez Almeida, J., \& Lites, B. W. 2000, ApJ, 532, 1215

Schlichenmaier, R., \& Collados, M. 2002, A\&A, 381, 668

Schüssler, M. 2001, in Advanced solar polarimetry: Theory, observation and instrumentetion, ed. M. Sigwarth, ASP Conf. Ser., 236,343

Sheminova, V. A. 2002, Kinematika Fiz. Nebesn. Tel, in press

Sigwarth, M. 2001, ApJ, 563, 1031

Sigwarth, M., Balasubramaniam, K. S., Knölker, M., \& Schmidt, W. 1999, A\&A, 349, 941

Socas-Navarro, H., López Ariste, A., \& Lites, B. W. 2001, ApJ, 553, 949

Socas-Navarro, H., \& Sánchez Almeida, J. 2002, ApJ, 565, 1323

Socas-Navarro, H., \& Sánchez Almeida, J. 2003, ApJ, submitted

Solanki, S. K. 1993, Space Sci. Rev., 63, 1

Solanki, S. K. 1994, in Infrared Sol. Phys., ed. D. M. Rabin, J. T. Jefferies, \& C. Lindsey (Dordrech: Kluwer), 236, 393

Solanki, S. K., Keller, C., \& Stenflo, J. O. 1987, A\&A, 188, 183 
Solanki, S. K., Rüedi, I., \& Livingston, W. 1992, A\&A, 263, 312

Solanki, S. K., \& Stenflo, J. O. 1984, A\&A, 140, 185

Solanki, S. K., Zufferey, D., Lin, H., Rüedi, I., \& Kuhn, J. R. 1996, A\&A, 310, L33

Spruit, H. C. 1979, Sol. Phys., 61, 363

Spruit, H. C., \& Zweibel, E. G. 1979, Sol. Phys., 62, 15

Stenflo, J. O. 1973, Sol. Phys., 32, 41

Stenflo, J. O. 1994, Solar Magnetic Fields. Polarized Radiation Diagnostics (Dordrecht: Kluwer)

Stenflo, J. O. 1999, in Solar Polarization II, ed. K. N. Nagendra, \& J. O. Stenflo (Dordrecht: Kluwer), 243, 1
Stenflo, J. O., Solanki, S. K., \& Harvey, J. W. 1987, A\&A, 173, 167 del Toro Iniesta, J. C., Bellot Rubio, L. R., \& Collados, M. 2001, ApJ, 549, L139

Trujillo Bueno, J. 2003, in Modelling of Stellar Atmospheres, IAU Symp. 210, ed. N. E. Piskunov, N. W. Weiss, \& D. E. Gray, ASP Conf. Ser., in press

Venkatakrishnan, P. 1986, Nature, 322, 156

Webb, A. R., \& Roberts, B. 1978, Sol. Phys., 59, 249

Zirin, H. 1985, Aust. J. Phys., 38, 961

Zirin, H. 1993, in The Magnetic and Velocity Fields of Solar Active Regions, ed. H. Zirin, G. Ai, \& H. Wand, ASP Conf. Ser., 46, 215 